\title{
theoretical and applied fracture mechanics
}

ELSEVIER

\section{Mesofracture mechanics: a necessary link}

\author{
G.C. Sih ${ }^{\text {a,b,c,* }, \text { B. Liu }}{ }^{\mathrm{c}}$ \\ a Department of Mechanical Engineering and Mechanics, Lehigh University, Bethlehem, PA 18015, USA \\ b Institute of Mechanics, Chinese Academy of Sciences, Beijing 100080, China \\ ${ }^{\mathrm{c}}$ Institute of Engineering Mechanics, Hebei University of Technology, Tianjin 300130, China
}

\begin{abstract}
Classical fracture mechanics is based on the premise that small scale features could be averaged to give a larger scale property such that the assumption of material homogeneity would hold. Involvement of the material microstructure, however, necessitates different characteristic lengths for describing different geometric features. Macroscopic parameters could not be freely exchanged with those at the microscopic scale level. Such a practice could cause misinterpretation of test data. Ambiguities arising from the lack of a more precise range of limitations for the definitions of physical parameters are discussed in connection with material length scales. Physical events overlooked between the macroscopic and microscopic scale could be the link that is needed to bridge the gap. The classical models for the creation of free surface for a liquid and solid are oversimplified. They consider only the translational motion of individual atoms. Movements of groups or clusters of molecules deserve attention. Multiscale cracking behavior also requires the distinction of material damage involving at least two different scales in a single simulation. In this connection, special attention should be given to the use of asymptotic solution in contrast to the full field solution when applying fracture criteria. The former may leave out detail features that would have otherwise been included by the latter. Illustrations are provided for predicting the crack initiation sites of piezoceramics. No definite conclusions can be drawn from the atomistic simulation models such as those used in molecular dynamics until the non-equilibrium boundary conditions can be better understood. The specification of strain rates and temperatures should be synchronized as the specimen size is reduced to microns. Many of the results obtained at the atomic scale should be first identified with those at the mesoscale before they are assumed to be connected with macroscopic observations. Hopefully, "mesofracture mechanics" could serve as the link to bring macrofracture mechanics closer to microfracture mechanics. (C) 2001 Published by Elsevier Science Ltd.
\end{abstract}

...reducing every problem to its smallest and simplest components so that it may be more easily understood and then solved. ...meddling in arcane disciplines where the mantra is "learn more and more about less and less.

Descartes' school of thought

\footnotetext{
${ }^{*}$ Corresponding author. Present address: School of Advanced Science and Technology, Xi'an Jiaotong University, Xi'an Shaanxi 710049, China. Fax: +86-29-2668028.

E-mail address: gcs@xjtu.edu.cn (G.C. Sih).
}

\section{Introduction}

Fracture mechanics was born out of necessity during the post-World War II period when large size structures such as passenger air transports, ships, storage tanks, etc, were cracking unexpectedly for no obvious reasons. To fulfill the need for 
failure prevention, the discipline was developed as a methodology rather than a science. Even though the basic mechanisms of fracture were never understood, fracture control was successfully implemented. This can be evidenced in the FAA guidelines for certifying aircraft structural components and sub-assemblies, NRC regulations for nuclear reactor components (ASME Codes Sections III and XI), welding codes for ship structure, etc. Much of the success depended on the transfer of test data from specimens of the order of $10 \mathrm{~cm}$ in lineal dimension to the design of full scale structures that are several orders of magnitude larger. Often, a prototype model intermediate in size was needed to fill in the gap. In retrospect, the experience and knowledge gained at that time should be more precisely identified with macrofracture mechanics and structure-fracture mechanics so as to emphasize the scale aspect of the problem.

The transfer of information from macrosize specimens to full or median size structures relied on "system homogeneity" that makes possible a direct link between the local parameter and the crack tip stress intensity factor, the critical value of which corresponds to global instability. This led to the concept of "fracture toughness" which in a strict sense applies only to a homogeneous material at the macroscopic scale. That is the specimen size had to be sufficiently large in comparison with the characteristic length of the material microstructure.

The fundamental nature of the foregoing remarks cannot be overemphasized when the concept of fracture toughness endorsed of ASTM was applied to composite materials that are no longer homogeneous. The classical critical energy release rate or stress intensity factor became dependent on the fiber orientation, stacking sequence, and so on. The interaction effect of load and geometry can no longer be sorted out from the material parameter that fracture toughness did for the homogeneous material. Being a tailor-made material for serving a specific mission, it would be futile to standardize composite material testing because this would defeat the purpose of its very own existence.

Applications of macrofracture mechanics to examine the failure of functional materials $[1,2]$ used for electric devices have been problematic and frustrating after recognizing that the structural device is many times smaller than the standard ASTM fracture test specimens. It makes little sense to apply the data collected from a large structure as an average to analyze the behavior of a portion of its constituents. Preservation of the degree of homogeneity between the structure and specimen has been violated. To put it in short terms the data transfer process cannot be reciprocated.

It is now more than 35 years since the first International Conference of Fracture (ICF) was held in Sendai, Japan. The objective then was for the dislocationists and continuum mechanicists to interact with the anticipation that the micro- and macroapproach of fracture could join together. The 10th anniversary of ICF held every four years will soon take place in Hawaii, December 2001. With the Pandora's box of fracture mechanics open, it is fair to say that the problem of fracture has been found to be far more complex than it was envisioned in 1965. High power resolution election microscopes continue to supply detail features of the material structure that have escaped the attention of the theoreticians. The micro/macro gap is overflowing with information; there appears the need to refine the scale divisions. The range in characteristic size should perhaps include a segment for mesoscopic events in between those for the microscopic and macroscopic scales. Even nanostructure materials are being considered as a challenge for the 21th century. While the origin of failure will probably never be found, it is still helpful to learn "what is not". For decades, dislocations were considered to be the source of failure initiation. However, they are too large to prevail at the nanoscale. Does this mean that nanomaterial will never fail?

This work will take the opportunity not only to point out the intricacies of the free surface creation process being fundamental to fracture but also cautions not to overlimit the application of continuum mechanics theories to specific scale size. Keep in mind that continuum mechanics is free of inherent characteristic length as long as continuity is satisfied. What should be more carefully observed is the use of asymptotic and full field solution when analyzing fracture sites. The exclusion 
of higher-order terms in a limiting process may exclude certain detail features that were overlooked previously and could be relevant to multiscale fracture problems. Physical understanding of the fracture process should take precedence even when analytical and experimental results seem to agree.

\section{Mechanisms of free surface creation}

Failure by fracture rests on the notion that free surface area is created when the prevailing driving force exceeds that which holds the atoms together. The threshold for permanent separation of the atoms has been identified with the cohesive bond strength. A specific surface energy is thus defined to represent the energy required to create a unit free surface area.

\subsection{Equilibrium and non-equilibrium free surface}

Hypothetically speaking, free surface may exist only in a perfect vacuum where all disturbances are absent. Equilibrium mechanics defines a free surface by the traction free condition

$\stackrel{n}{T_{i}}=\sigma_{j i} n_{j} \rightarrow 0$,

where $\stackrel{n}{i}_{i}$ stand for the tractions on the surface with the outward unit normal vector components $n_{j}$. The stresses are $\sigma_{i j}$.

In a non-equilibrium theory of thermomechanics $[3,4]$, the free surface still corresponds to the traction free condition but the surface may undergo oscillation depending on the characteristic length parameter $\mathrm{d} V / \mathrm{d} A$ which represents the differential rate change of volume $V$ with surface area $A$. The oscillatory character of the free surface may be expressed as $[3,4]$

$\stackrel{n}{T_{i}}=\sigma_{j i} n_{j}+\left(\rho_{0} \ddot{u}_{i}-\mathrm{F}_{i}\right)\left(\frac{\mathrm{d} V}{\mathrm{~d} A}\right)_{n} \rightarrow 0$,

where $u_{i}$ are the displacement components, $F_{i}$ the body forces and $\rho_{0}$ is the mass density. Dot denotes time differentiation and hence $\rho_{0} \ddot{u}_{i}$ stands for the inertia which tends to increase with decreasing scale size. The product $\rho_{0} \ddot{u}_{i}(\mathrm{~d} V / \mathrm{d} A)_{n}$ is not negli- gible. Rippling of a liquid surface is not an uncommon phenomenon.

The flow of air next to the surface of a solid aluminum cylinder stretched at a strain rate of $1.27 \times 10^{-3} \mathrm{~s}^{-1}$ has been studied in [5]. Next to the air/solid interphase, both the thermal and mechanical responses are highly transient in character. For an open thermodynamic system, there prevails only bi-phase problems where the conditions on the interphase are determined rather than pre-assigned as it is necessary in boundary value problems for closed thermodynamics systems. To be kept in mind is that classical mechanics deals exclusively with closed systems where the concept of a "free surface" follows the mathematical condition of Eq. (1) rather than what takes place in a test. The deviation from reality could be of firstorder magnitude when the scale level of investigation is reduced to micro- and nanoscale levels.

\subsection{Breaking of atomic bond strength}

Suppose that a fracture surface could be ideally modeled by two adjacent planes of atoms separated by a distance $a_{0}$ of the order of $10^{-8} \mathrm{~cm}$ as shown in Fig. 1(a). The uniform local pulling stresses $\sigma$ are resisted by the bonding forces between the atoms with a threshold quantified by the bond strength $\sigma_{\mathrm{c}}$. The corresponding displacement is $x_{\mathrm{c}}$ beyond which the atoms would no longer return to their original positions. Lacking a knowledge of how $\sigma$ would vary with $x$, a linear relationship [6] in Fig. 1(b) is assumed. The work done per unit area for breaking the atomic bonds can be calculated as

$2 \gamma_{\mathrm{c}}=\int_{0}^{x_{\mathrm{c}}} \sigma(x) \mathrm{d} x=\frac{1}{2} \sigma_{\mathrm{c}} x_{\mathrm{c}}$.

If $x_{\mathrm{c}} / a_{0}$ is defined as the cohesive strain, then a linear stress-strain relation gives

$x_{\mathrm{c}}=a_{0}\left(\frac{\sigma_{\mathrm{c}}}{e_{0}}\right)$,

with $e_{0}$ being a measure of the stiffness response. Eliminating $x_{\mathrm{c}}$ between Eqs. (3) and (4), there results the cohesive bond stress 


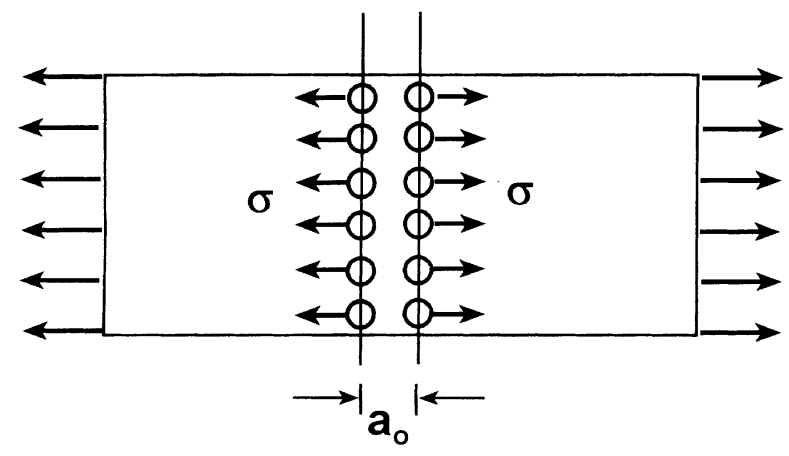

(a)

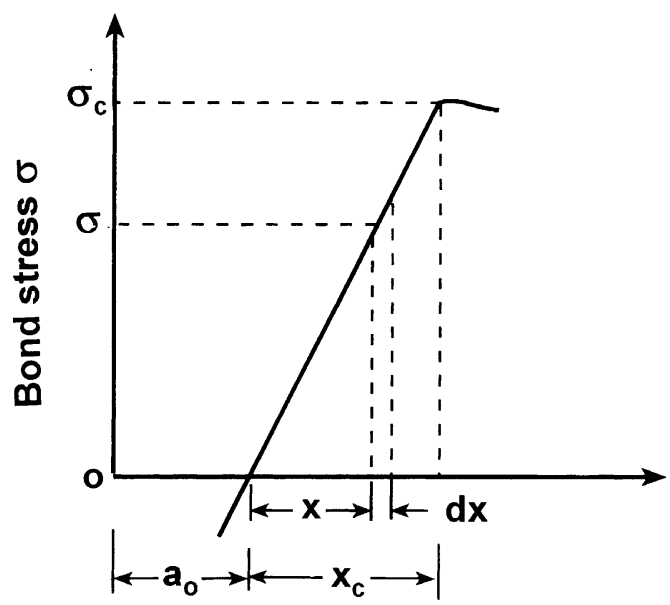

(b)

\section{Distance $\mathrm{x}$}

Fig. 1. Adjacent planes of atoms under stress: (a) atomic planes; (b) bond stress versus distance.

$\sigma_{\mathrm{c}}=2 \sqrt{\frac{e_{0}}{a_{0}}} \sqrt{\gamma_{\mathrm{c}}}$.

This result differs from that in ordinary textbooks by a factor of 2 owing to the difference of using a linear stress-strain curve in Fig. 1(b) and a sine curve, the assumption of which is also arbitrary. A replacement of $4 e_{0}$ by $E_{0}$ puts Eq. (5) in textbook form as $\sigma_{\mathrm{c}}=\sqrt{E_{0} \gamma_{\mathrm{c}} / a_{0}}$. Here, Young's modulus is $E_{0}$. The cohesive bond strength $\sigma_{\mathrm{c}}$ has been referred to as the "ideal" strength of a solid. It is approximately two orders of magnitude higher than the ultimate strength $\sigma_{\mathrm{u}}$ measured from specimens at the macroscopic scale.

For an order of magnitude comparison, consider drawn silica glass [7] with $E_{0} / \sigma_{\mathrm{u}}=7.35$ and $E_{0}=73.5 \mathrm{GPa}$. The atoms or molecules are about $3 \times 10^{-8} \mathrm{~cm}$ distance apart. Using the measured values of $\sigma_{\mathrm{u}}$ [7] instead of the ideal strength $\sigma_{\mathrm{c}}$, Eq. (5) becomes $\gamma_{\mathrm{c}}=a_{0}\left(\sigma_{\mathrm{u}} / E_{0}\right) \sigma_{\mathrm{u}}$. This gives $\gamma_{\mathrm{c}}=392 \mathrm{~Pa} \mathrm{~m}$. Free interchanges of $e_{0}$ with $E_{0}$ and $\sigma_{\mathrm{c}}$ with $\sigma_{\mathrm{u}}$ at the different scale levels are common and will be discussed subsequently in connection with scale shifting [8].

\subsection{Surface tension of a liquid}

A liquid can minimize its surface energy by adopting a shape of minimum surface area as if enclosed in a tensile skin. The process involves bringing internal particles (or molecules) from the interior to the surface. The increases in the number of particles cause stretching of the skin. The resulting constant tension is known as "surface tension" $\gamma_{s}$ in contrast to tensile force between particles, Fig. 1(a). The creation of new free surface by stretching is illustrated in Fig. 2.

The difference between the ideal and measured strength of glass fibers has been attributed to the presence of surface flaws [9] that were not considered in Eq. (5). A crack growth instability model was proposed; it consists of a narrow ellipse with surface area $4 a \cdot 1$ with unity assumed as the thickness. With a 10:1 aspect ratio of the ellipse, the area of the upper and lower surface is assumed to be evenly divided. For the mathematical model to be consistent with the physical model [9], the periphery of the narrow elliptical cavity was visualized to expand confocally and collapse like a soap bubble. Recall that the collapse condition for a spherical bubble with radius $R$ under uniform pressure $p$ is given by $p R=2 \gamma_{\mathrm{s}}$, where $\gamma_{\mathrm{s}}$ is the surface tension. The equivalent expression for a narrow elliptical cavity in Fig. 3(a) has been derived [6]:

$\frac{p a \sqrt{a / \rho}}{K(k)}=\frac{4}{\pi} \gamma_{s}, \quad k^{2}=1-(\rho / a)$, 


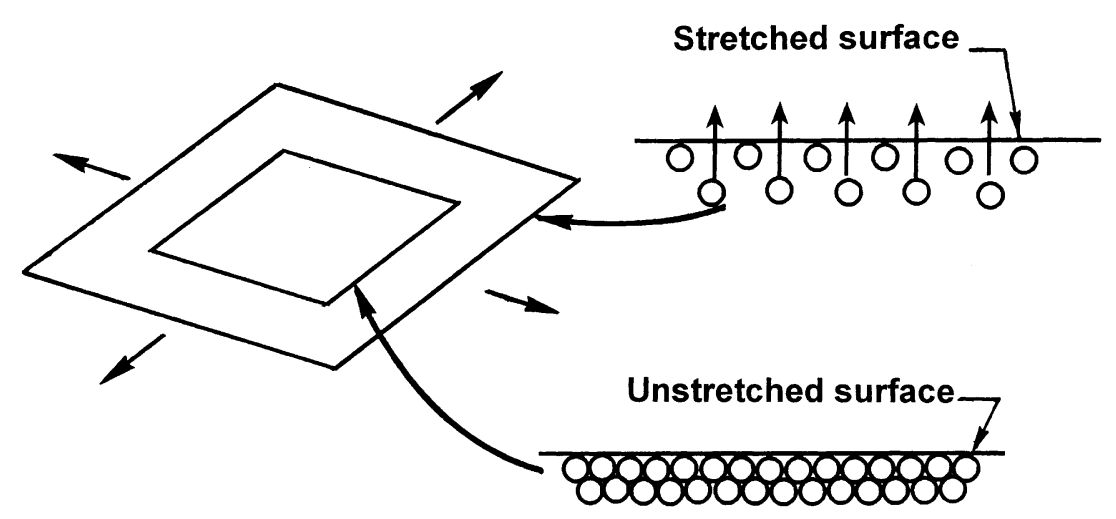

Fig. 2. Schematic of free surface creation: moving particles to surface.
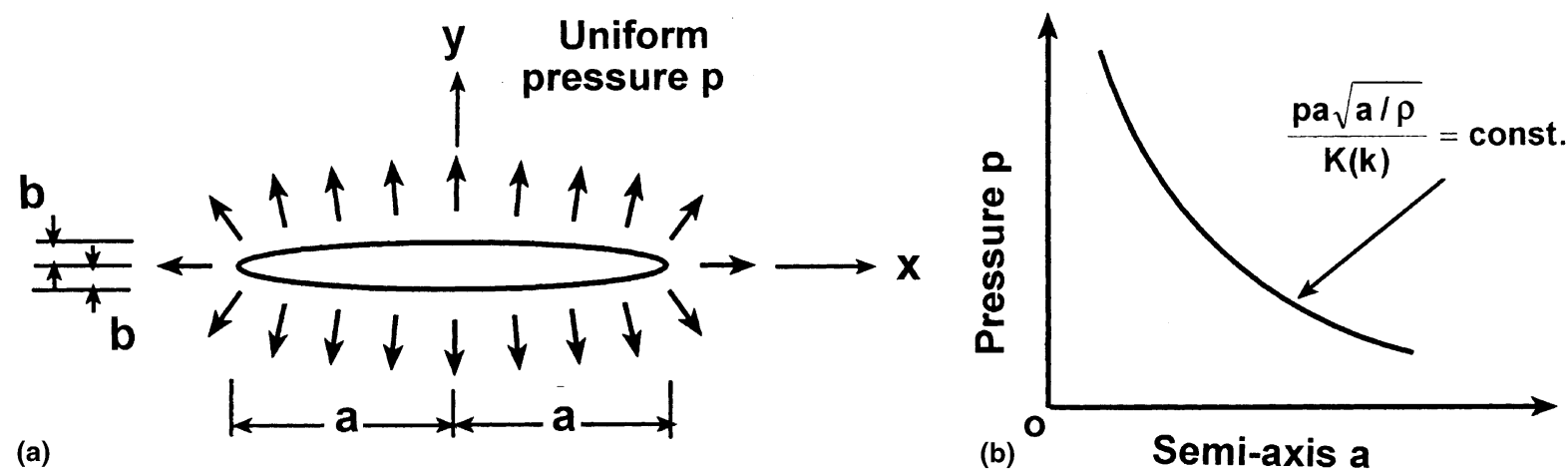

Fig. 3. Stretching behavior of narrow elliptical cavity: (a) narrow ellipse; (b) variations of $p$ with $a$.

where $\rho=b^{2} a$ is the radius of curvature of a blunted crack front and $K(k)$ is the complete elliptical integral of the first kind with argument $k$. Fig. 3(b) displays the variations of $p$ given by Eq. (6). The dominance of $p^{2} a=$ const. is clear. This is reminiscent of the $\sigma^{2} a=$ const. relation for the Mode I stress intensity factor in linear elastic fracture mechanics (LEFM). Although the decay of the applied stress or pressure with defect (crack) length has the same trend, the free surface creation process differs in basic character: one is bond breaking for the solid and the other is thin film stretching for the liquid.

Consistent with the surface tension model of a narrow ellipse, silica glass fibers were heated from 745 to $1110^{\circ} \mathrm{C}$ [9] so that the glass would become viscous and flow. A reported value of $437.5 \mathrm{~Pa} \mathrm{~m}$ was identified with $\gamma_{\mathrm{s}}$; it was extrapolated to 524.5 $\mathrm{Pa} \mathrm{m}$ at $15^{\circ} \mathrm{C}$ with the aim to explain the fracture behavior of glass in the solid state. Whether the extrapolation would indeed change the free surface creation mechanism from surface tension to bond breaking is doubtful. Moreover, the mechanisms of free surface creation may depend on the network structure of the glass that determines the extent of viscous flow. Note the orderly network of silica in the form of quartz in Fig. 4(a) as compared with the disorderly form for glass in Fig. 4(b). The network formation would necessarily impose constraints on the movements of the molecules and hence on the process of free surface creation.

\subsection{Amorphous material behavior}

When glass is heated to a viscous state, it will neither behave completely as a liquid nor as a solid where the atoms or molecules would only 


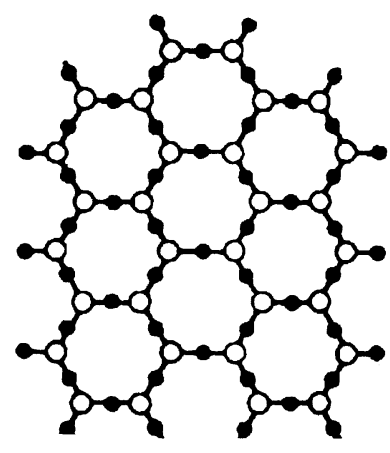

(a)

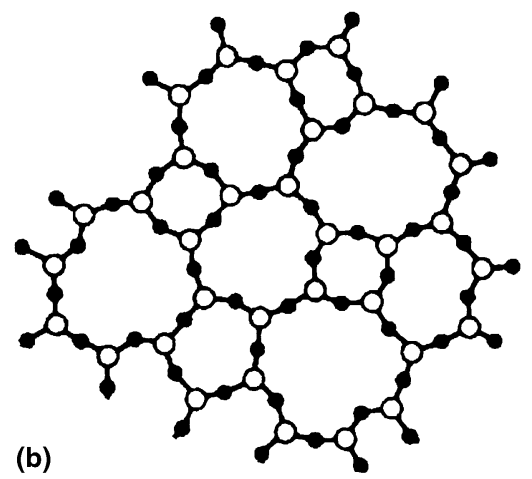

(b)

Fig. 4. Network structure of silica (orderly and disorderly): (a) quartz crystal (orderly); (b) glass (disorderly).

move in translation. However, there cannot be a complete distinction between these concepts. Viscosity could alter the network such that the molecules could move in groups undergoing translation as well as rotation. The classical definitions of surface tension and bond breaking referred to individual molecules may not hold. To emphasize that molecules could move in clusters such that free surface is created by translational and rotational motions of these clusters from inside to the free surface is illustrated in Fig. 5. It is necessary to examine the "mesoscopic" range. Not individual molecules but groups of them are the key players. The same holds for dislocations in relation to formulating elastoplastic theories where the essential features of plasticity are mesoscopical in character. The interest would be dislocation groups rather than individual dislocations [10].

Moreover, molecules near a surface are less constrained than those inside. They are more likely to be distorted by shear. Referring to Fig. 6(a), antisymmetry of the stress tensor $\tau_{i j} \neq \tau_{j i}$ in the boundary condition $T_{i}=\tau_{j i} n_{j}$ defined in Eq. (2) gives a moment, $M=\tau_{y x}-\tau_{x y}$. Consider only the $x$ - and $y$-components for illustration, the in-plane shear stress components are:

$$
\begin{aligned}
& \tau_{x y}=\sigma_{x y}+\rho_{0} \ddot{u}_{y}\left(\frac{\mathrm{d} V}{\mathrm{~d} A}\right)_{x}, \\
& \tau_{y x}=\sigma_{y x}+\rho_{0} \ddot{u}_{x}\left(\frac{\mathrm{d} V}{\mathrm{~d} A}\right)_{y} .
\end{aligned}
$$

Rotary motion is thus enhanced near the surface, particularly when the inertia terms in (7) are appreciable. Remember that a normal macrostrain rate may correspond to extremely high rates at the microscale or atomic scale. It can be as high as $10^{6}-10^{12} \mathrm{~s}^{-1}$. This was pointed out in $[11,12]$ with reference to the application of the atom embedded method (EAM). In this sense, local inertia effects represented by the terms $\rho_{0} \ddot{u}_{x}$

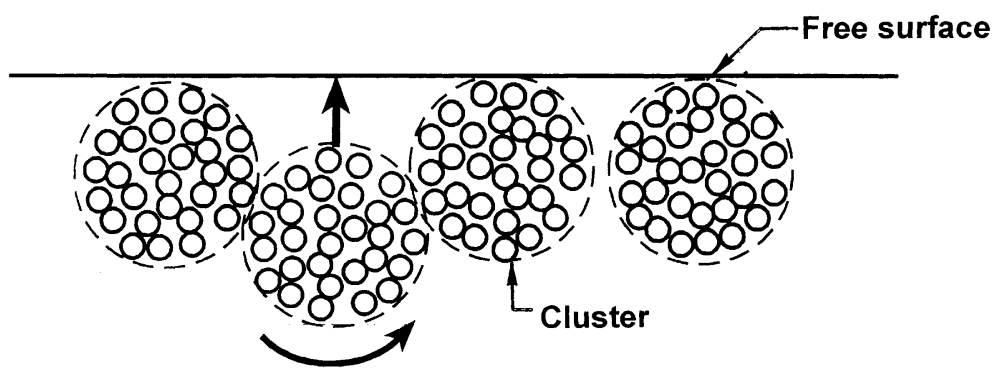

Fig. 5. Motion of a cluster of molecules. 


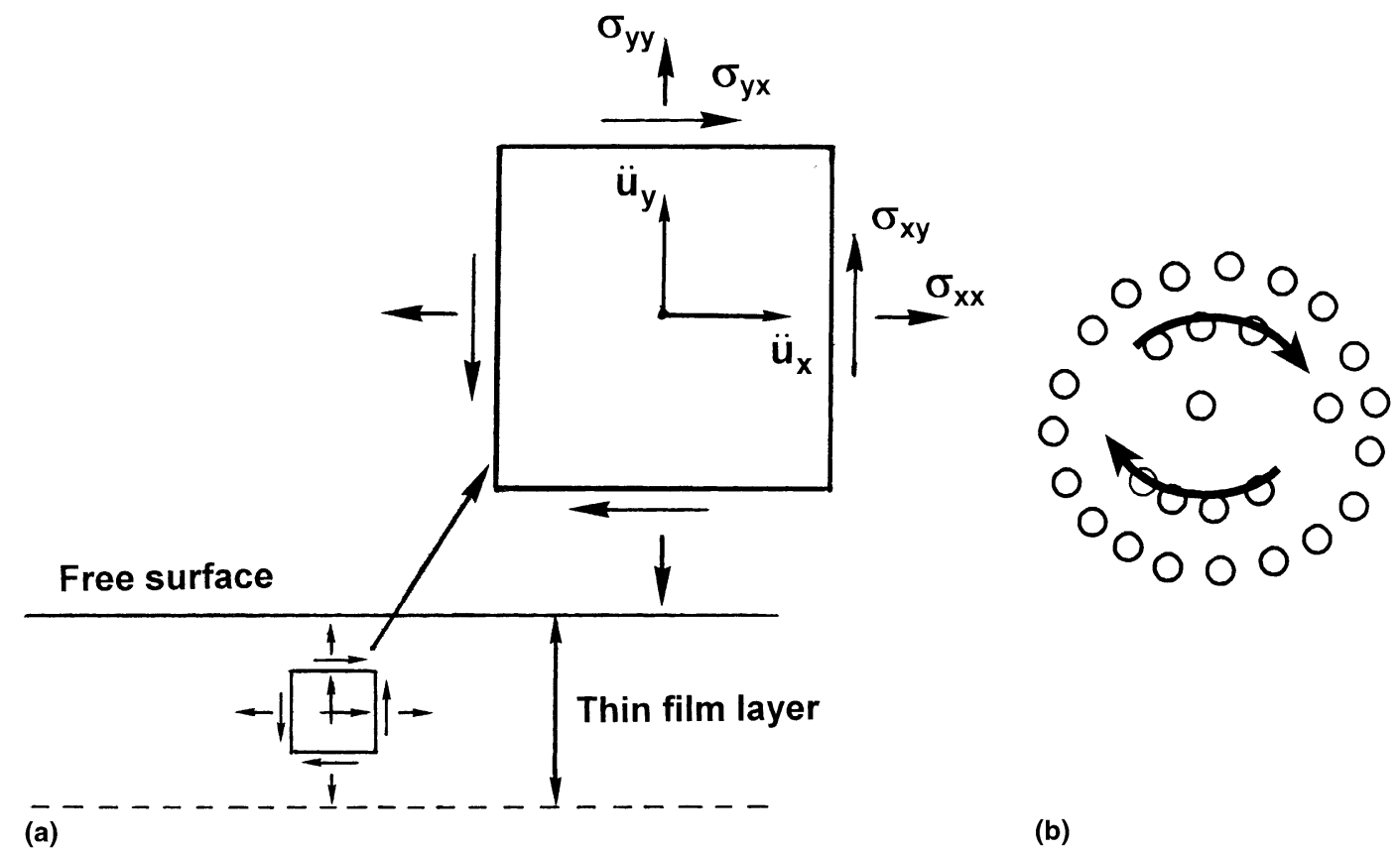

Fig. 6. Rotational action in free surface layer: (a) surface layer; (b) vortex action.

and $\rho_{0} \ddot{u}_{y}$ could be large even though $(\mathrm{d} V / \mathrm{d} A)_{j}$, $j=x, y$ may be small. Vorticity may thus be present, Fig. 6(b). Indeed, this was observed in [13-15] at the scale level of inhomogeneities such as inclusions, grains, etc. They occur in the mesoscale region within which the action of shear + rotation is significant. These observations would normally be bypassed because attention in the past have been focused primarily on the macro- and microscales.

Recent advances in nanomaterials and nanoliquids have also led to the speculation that atoms may group as clusters (Fig. 5) in situations where the bonding forces are highly non-uniform. This may be created by chemical reactions and/or powerful laser beams to stir up the atoms and create differences in the bonding. Those with stronger bonding would self-organize themselves and form a crust trapping the ones with weaker bonding inside. Individual units of nanometer size could presumably be stabilized without merging into one another. Note that one nanometer is onebillionth of a meter. It is a linear distance spanning approximately 10 atomic diameters.

\section{Void or cavity creation mechanism}

The creation of voids or cavities in solids is caused by material microstructure inhomogeneity and hydrostatic tension. The sites of nucleation correspond to local regions where volume change would dominate in relation to shape change. The terminology "notch embrittlement" applies to the situation illustrated in Fig. 7(a). Grains in a polycrystal are highly inhomogeneous. The crack front would seek out the stronger grains and rest against them. The local hydrostatic tensile stress field pulls the cluster of grains evenly on all sides, dissipating relatively little energy in distortion. Under increasing load, the local hydrostatic tension would break the weaker grains which are always situated at a finite distance away from the crack front. For stretched thin metal foils, voids have been observed to occur ahead of a main crack as bright spots where light is projected to the back of the foil, Fig. 7(b). Crack growth takes place due to coalescence of these voids; it is a discrete rather than a continuous process assumed in idealized theoretical models. Such a behavior is more pro- 


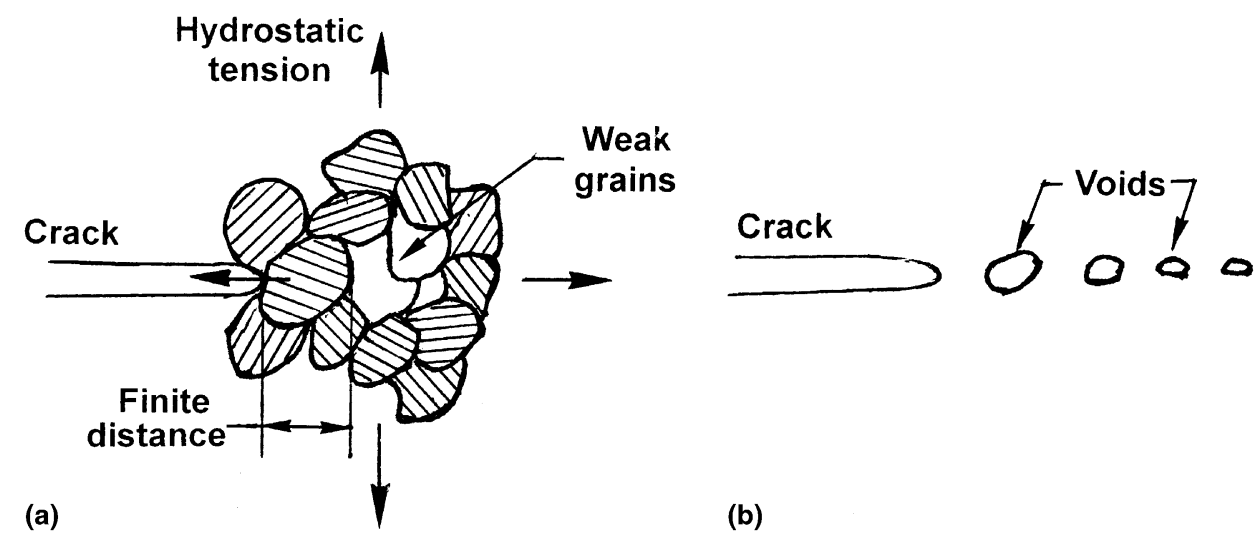

Fig. 7. Mechanism of void creation: (a) grain inhomogeneity; (b) coalescence of voids.

nounced in the ductile metal although grain cracking in ceramics prior to growth of the main crack is common. As a rule, microcracking occurs readily in brittle materials and can be quantified by acoustic emission counts.

\subsection{Volume energy density}

Consider a unit volume of material ahead of a macrocrack that is many times larger than the grain as shown in Fig. 8(a). A core region of radius $r_{0}$ is introduced as a scale length that separates the regions of macroscopic homogeneity from those of microscopic inhomogeneity. This is illustrated in Fig. 8(a) with an enlarged view of the volume element in Fig. 8(b) in two dimensions. The parameter $r_{0}$ also marks the cutoff distance for stress, strain energy density, etc., that would tend to be- come unbounded as $r$ tends to zero. Here, $r$ is limited to $r_{0}$ and is no smaller.

More specifically, let the energy stored in a differential volume $\mathrm{d} V$ be written as [16-18]

$\frac{\mathrm{d} W}{\mathrm{~d} V}=\frac{S}{r}$,

in which $S$ is known as the volume energy density factor. It represents the area under the $\mathrm{d} W / \mathrm{d} V$ versus $r$ curve, Fig. 9 and stands for the energy release if the crack extends an amount $r-r_{0}$. The distance $r_{0}$ should be kept very small $r_{0} / r \ll 1$ such that the shaded area ABCD in Fig. 9 is negligible when compared with $S$. Otherwise, ABCD would contribute to crack growth overshoot, an effect that is not insignificant $[19,20]$. The critical value $(\mathrm{d} W / \mathrm{d} V)_{\mathrm{c}}$ corresponds to the onset of stable or unstable crack growth depending on the conditions that

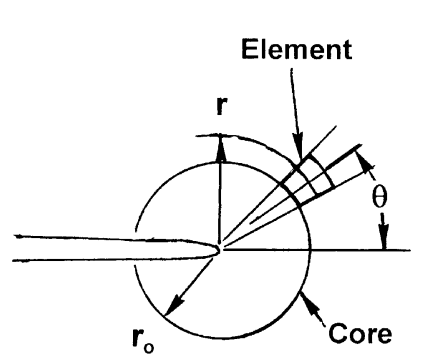

(a)

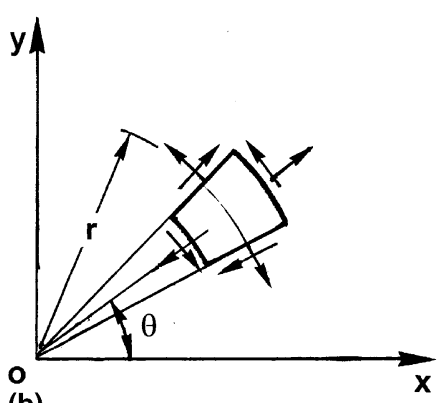

(b)

Fig. 8. Schematic of near tip volume element: (a) core region; (b) near tip element. 


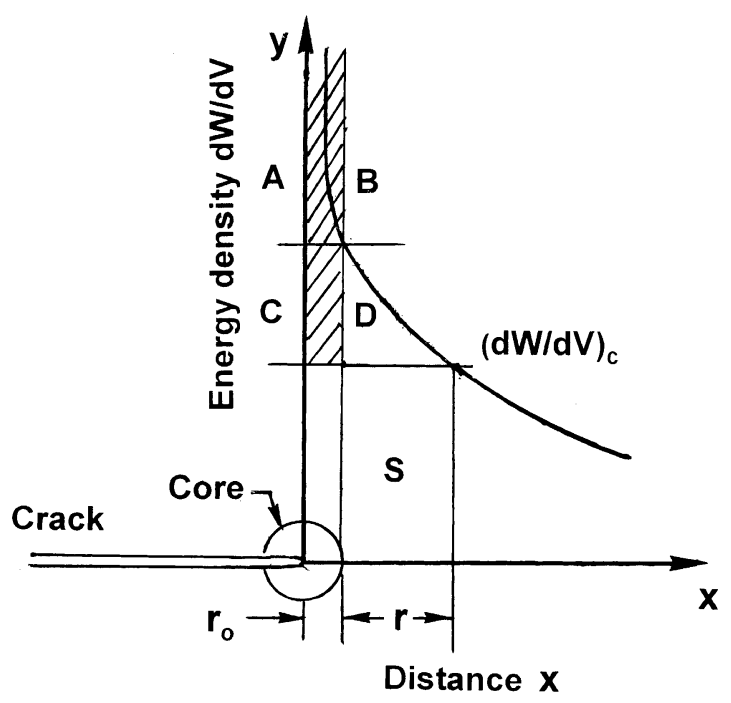

Fig. 9. Volume energy decay with distance.

Stable growth: $\quad S<S_{\mathrm{c}}$,

Unstable growth: $S>S_{\text {c }}$.

In fact, $(\mathrm{d} W / \mathrm{d} V)_{\mathrm{c}}$ is the area under the uniaxial true stress and true strain curve:

$$
\left(\frac{\mathrm{d} W}{\mathrm{~d} V}\right)_{\mathrm{c}}=\int_{0}^{\varepsilon_{\mathrm{c}}} \sigma(\varepsilon) \mathrm{d} \varepsilon
$$

where $\varepsilon_{\mathrm{c}}$ is the critical strain.

The ASTM valid plane strain toughness value $K_{\mathrm{IC}}$ is related to $S_{\mathrm{c}}$ by the relation

$S_{\mathrm{c}}=\frac{(1+v)(1-2 v) K_{\mathrm{IC}}^{2}}{2 \pi E_{0}}$.

Even though the $1 / r$ decay for $\mathrm{d} W / \mathrm{d} V$ coincides with the $1 / \sqrt{r}$ stress singularity solution obtained from linear elasticity, Eq. (8) remains valid for elastoplastic materials in general. In addition to the definition for $S$, Eq. (8) would give finite energy upon integration around the crack tip, since the differential element area depends on $r$. The volume energy density has been referred to as the absorbed specific energy in [21-23] and used as a parameter for ranking the resistance of the material to fracture. It was adopted in the Hungarian codes and used for fracture testing.

\subsection{Inherent coupling of distortion and dilation}

No difficulties would be met with when using the volume energy density as a fracture criterion [17]. Remember that the specific surface energy $\gamma_{c}$ was unable to characterize the different viscous states of glass as it flows and solidifies. The stationary values of $\mathrm{d} W / \mathrm{d} V$ can automatically account for the proportion of distortion (ductile behavior) and dilatation (brittle behavior). When non-linearity is present, it is not possible to use linear elasticity and divide $\mathrm{d} W / \mathrm{d} V$ into a part for dilatation

$$
\left(\frac{\mathrm{d} W}{\mathrm{~d} V}\right)_{\mathrm{v}}=\frac{1-2 v}{6 E_{0}}\left(\sigma_{x}+\sigma_{y}+\sigma_{z}\right)^{2},
$$

and another for distortion

$$
\begin{aligned}
\left(\frac{\mathrm{d} W}{\mathrm{~d} V}\right)_{\mathrm{d}}= & \frac{1+v}{6 E_{0}}\left[\left(\sigma_{x}-\sigma_{y}\right)^{2}+\left(\sigma_{y}-\sigma_{z}\right)^{2}\right. \\
& \left.+\left(\sigma_{z}-\sigma_{x}\right)^{2}+6 \sigma_{x y}^{2}\right] .
\end{aligned}
$$

Eqs. (12) and (13) apply to plane strains, where $\sigma_{z}=v\left(\sigma_{x}+\sigma_{y}\right)$ and $\sigma_{x z}=\sigma_{y z}=0$. With reference to the principal planes, $\sigma_{x}=\sigma_{1}, \sigma_{y}=\sigma_{2}$ and $\sigma_{x y}=0$. Plasticity being a non-linear theory, however, assumed linearity as an a priori by using Eq. (13) for the yield criterion (von Mises). The neglect of Eq. (12) ahead of the crack front is not justified because the hydrostatic tension is so high that it leads to void creation, Fig. 7(b). Classical plasticity theory tends to underestimate the state of affairs local to the crack $[24,25]$ by a large margin.

Additional insights may be gained by observing the differential change of volume $\mathrm{d} V$ which is relatively small if dilatation dominates, Fig. 10(a) and large if distortion dominates, Fig. 10(b). In plasticity, $\mathrm{d} V=0$ appears to be invoked as a rule rather than the exception. In general, the proportion of distortion to dilatation should be determined analytically or measured experimentally. In two dimensions, $\mathrm{d} W / \mathrm{d} V$ is a function of both $r$ and $\theta$ and may be taken to determine the sites of the relative minimum and maximum with the interpretation that 


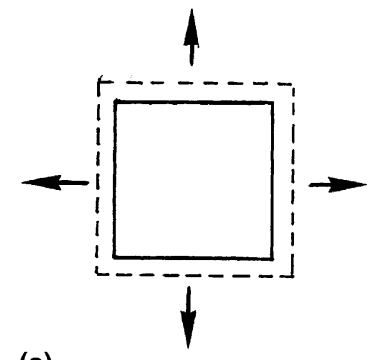

(a)
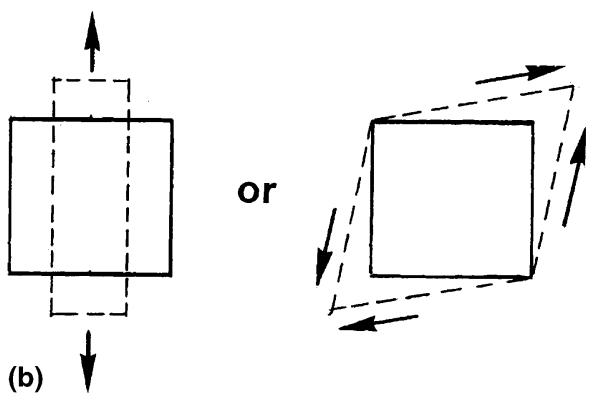

Fig. 10. Relative magnitude of $\mathrm{d} W / \mathrm{d} V$ for dilatation and distortion: (a) dilatation ( $\mathrm{d} W / \mathrm{d} V \ll)$; (b) distortion ( $\mathrm{d} W / \mathrm{d} V \gg$ ).

Relative minimum of $\frac{\mathrm{d} W}{\mathrm{~d} V}$ :

Dilation $>$ Distortion,

Relative maximum of $\frac{\mathrm{d} W}{\mathrm{~d} V}$ :

Distortion $>$ Dilation.

The conditions in Eqs. (14) could be checked explicitly for the linear case where the elasticity solution of a Mode I crack is readily available.

\subsection{Sites of yielding and fracture}

The Mode I crack behavior will be used for illustration. By substitution of the crack tip stresses [17]

$\sigma_{x}=\frac{K_{1}}{\sqrt{2 \pi r}} \cos \frac{\theta}{2}\left[1-\sin \frac{\theta}{2} \sin \frac{3 \theta}{2}\right]+\cdots$,

$\sigma_{y}=\frac{K_{1}}{\sqrt{2 \pi r}} \cos \frac{\theta}{2}\left[1+\sin \frac{\theta}{2} \sin \frac{3 \theta}{2}\right]+\cdots$,

$\tau_{x y}=\frac{K_{1}}{\sqrt{2 \pi r}} \cos \frac{\theta}{2} \sin \frac{\theta}{2} \sin \frac{3 \theta}{2}+\cdots$

into $\mathrm{d} W / \mathrm{d} V$ for plane strain, it is found that

$\frac{\mathrm{d} W}{\mathrm{~d} V}=\frac{K_{1}^{2}}{16 \pi \mu r}[(3-4 v-\cos \theta)(1+\cos \theta)]$,

with $2 \mu=E_{0} /(1+v)$ being the shear modulus of elasticity. Instead of having both $r$ and $\theta$ as variables, consider $r=r_{0}$ as a constant and seek only for the variations of $\mathrm{d} W / \mathrm{d} V$ with $\theta$. Taking $\partial(\mathrm{d} W / \mathrm{d} V) / \partial \theta=0$, two possible solutions are found: $\theta_{0}=0, \quad \cos \theta_{\mathrm{p}}=1-2 v$.

These results can be inserted into the second derivatives of $\mathrm{d} W / \mathrm{d} V$ with respect to $\theta$ and show that the first and second angles $\theta_{0}$ and $\theta_{\mathrm{p}}$ are summarized in Figs. 11(a) and (b).

Substituting Eqs. (15) into Eqs. (12) and (13), gives for $\theta_{0}=0$ the results

$r_{0}\left(\frac{\mathrm{d} W}{\mathrm{~d} V}\right)_{\mathrm{v}}=\frac{2 K_{1}^{2}}{3 \pi E_{0}}(1-2 v)(1+v)^{2}$,

$r_{0}\left(\frac{\mathrm{d} W}{\mathrm{~d} V}\right)_{\mathrm{d}}=\frac{K_{1}^{2}}{2 \pi E_{0}}(1+v)(1-2 v)$.

Eqs. (18) shows that

$\left(\frac{\mathrm{d} W}{\mathrm{~d} V}\right)_{\mathrm{v}}>\left(\frac{\mathrm{d} W}{\mathrm{~d} V}\right)_{\mathrm{d}}$ for $\theta_{0}=0$.

The crack is thus predicted to grow along the direction where dilatation is greater than distortion. Similarly, the second solution $\cos \theta_{\mathrm{p}}=1-2 v$ in Eq. (17) yields

$$
\begin{aligned}
& r_{0}\left(\frac{\mathrm{d} W}{\mathrm{~d} V}\right)_{\mathrm{v}}=\frac{K_{1}^{2}}{3 \pi E_{0}}(1+v)(1-v)(1-2 v), \\
& r_{0}\left(\frac{\mathrm{d} W}{\mathrm{~d} V}\right)_{\mathrm{d}}=\frac{K_{1}^{2}}{6 \pi E_{0}}\left(1-v^{2}\right)\left(1-v+4 v^{2}\right),
\end{aligned}
$$

where

$$
\left(\frac{\mathrm{d} W}{\mathrm{~d} V}\right)_{\mathrm{d}}>\left(\frac{\mathrm{d} W}{\mathrm{~d} V}\right)_{\mathrm{v}} \text { for } \theta_{\mathrm{p}}= \pm \cos ^{-1}(1-2 v) .
$$

Yield initiation is predicted to occur off to the sides of the crack, the direction of which depends on the 


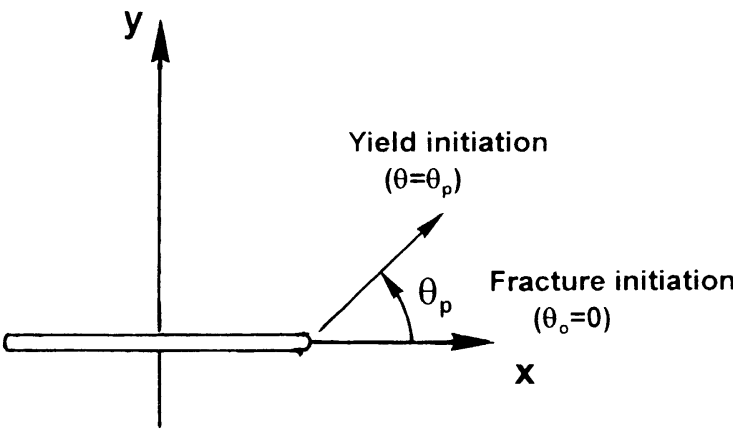

(a)

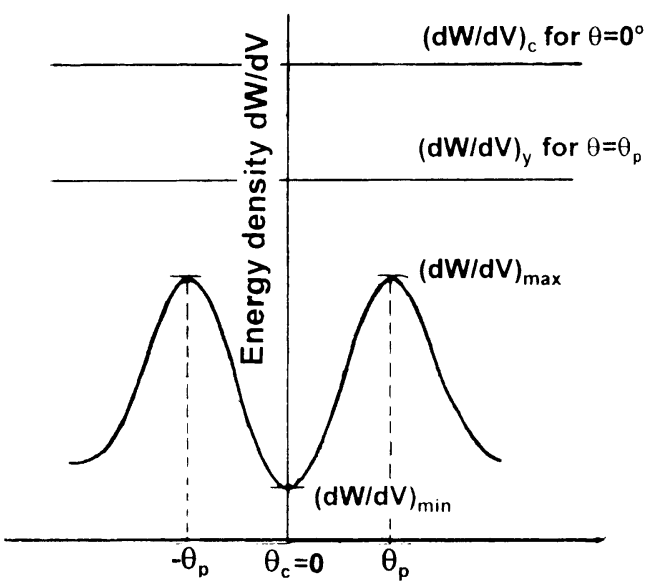

(b)

Angle $\theta$

Fig. 11. Direction of yield and fracture initiation: (a) Mode I crack; (b) angular variation.

Poisson ratio. For an incompressible material $v=1 / 2, \theta_{\mathrm{p}}=90^{\circ}$, i.e., the plastic enclaves would be directed normal to the crack. Eqs. (20) also show that $(\mathrm{d} W / \mathrm{d} V)_{\mathrm{d}}$ and $(\mathrm{d} W / \mathrm{d} V)_{\mathrm{v}}$ differ by $4-5$ times depending on $v$ and never by an order of magnitude. It is, therefore, not justified to neglect $(\mathrm{d} W / \mathrm{d} V)_{\mathrm{v}}$ in comparison with $(\mathrm{d} W / \mathrm{d} V)_{\mathrm{d}}$, an argument often adopted in plasticity to use the von Mises' yield criterion. To reiterate, the sites of yielding can be located simply by computing for $(\mathrm{d} W / \mathrm{d} V)_{\max }$. For regions not so close to the crack tip, the locations of $(\mathrm{d} W / \mathrm{d} V)_{\max }$ are found to be near the sites where the von Mises or effective stress is maximum.

Another piece of information follows directly from Fig. 11(b) which shows the angular variations of $\mathrm{d} W / \mathrm{d} V$. The threshold plateaus for yielding and fracture initiation correspond, respectively, to the lines $(\mathrm{d} W / \mathrm{d} V)_{\mathrm{y}}$ and $(\mathrm{d} W / \mathrm{d} V)_{\mathrm{c}}$, where $(\mathrm{d} W / \mathrm{d} V)_{\mathrm{c}}>(\mathrm{d} W / \mathrm{d} V)_{\mathrm{y}}$. As the load is raised, the curve $\mathrm{d} W / \mathrm{d} V$ would elevate and meet with the threshold lines at $\theta_{\mathrm{p}}$ for $(\mathrm{d} W / \mathrm{d} V)_{\max }$ and $\theta_{\mathrm{c}}=0^{\circ}$ for $(\mathrm{d} W / \mathrm{d} V)_{\min }$. The former would always precede the latter. That is yielding will always first occur off to the side of the main crack prior to crack growth. At a given scale level, zones of yielding tend to surround the prospective path of crack where stiffness has been built up. This can be observed from the formation of cup and cone after necking of a cylindrical bar [26]. Shear lips formed after dimpling of a finite thickness plate with a through crack is another example [27].

\subsection{Mutual dependence of volume and surface}

The notation $\mathrm{d} W / \mathrm{d} A$ was used in [28] to denote the "work per unit area for fracture propagation"; it is related to the Mode I plane strain stress intensity factor as

$K_{1}=\sqrt{\frac{E_{0}}{1-v^{2}} \frac{\mathrm{d} W}{\mathrm{~d} A}}$,

where $E_{0}$ and $v$ are, respectively, Young's modulus and the Poisson ratio. In Eq. (22), the differential increase in crack surface $\mathrm{d} A$ is straight ahead of the original crack plane.

In general, $\mathbf{d W} / \mathbf{d A}$ would be a vector quantity that could have three components $(\mathrm{d} W / \mathrm{d} A)_{j}$ identified with the coordinate axes $j=x, y, z$. Since a finite volume $V$ must necessarily be an integral part of the surface area $A$ and vice versa, $\mathrm{d} V / \mathrm{d} A$ is a non-zero quantity. It is also a vector having three components $j=x, y, z$ denoted by $(\mathrm{d} V / \mathrm{d} A)_{j}$. It follows that

$\left(\frac{\mathrm{d} W}{\mathrm{~d} A}\right)_{j}=\left(\frac{\mathrm{d} V}{\mathrm{~d} A}\right)_{j} \frac{\mathrm{d} W}{\mathrm{~d} V}, \quad j=x, y, z$,

where $\mathrm{d} W / \mathrm{d} V$ is a scalar. In the linear theory of elasticity, $(\mathrm{d} V / \mathrm{d} A)_{j}(j=x, y, z)$ are given by 


$$
\begin{aligned}
& \left(\frac{\mathrm{d} V}{\mathrm{~d} A}\right)_{x}=\frac{\varepsilon_{x}+\varepsilon_{y}+\varepsilon_{z}}{\varepsilon_{y}+\varepsilon_{z}} \ell_{0}, \ldots, \\
& \left(\frac{\mathrm{d} V}{\mathrm{~d} A}\right)_{z}=\frac{\varepsilon_{x}+\varepsilon_{y}+\varepsilon_{z}}{\varepsilon_{x}+\varepsilon_{y}} \ell_{0}
\end{aligned}
$$

where $\ell_{0}$ is the characteristic length of the element. It follows that

$$
\left|\frac{\mathrm{d} V}{\mathrm{~d} A}\right|=\sqrt{\left(\frac{\mathrm{d} V}{\mathrm{~d} A}\right)_{x}^{2}+\left(\frac{\mathrm{d} V}{\mathrm{~d} A}\right)_{y}^{2}+\left(\frac{\mathrm{d} V}{\mathrm{~d} A}\right)_{z}^{2}} \cdot \ell_{0} .
$$

It follows that

$$
\left|\frac{\mathrm{d} W}{\mathrm{~d} A}\right|=\left|\frac{\mathrm{d} V}{\mathrm{~d} A}\right| \frac{\mathrm{d} W}{\mathrm{~d} V} .
$$

Consider Mode I loading with $K_{1}=\sigma \sqrt{\pi a}$, where $\sigma$ is the remote loading applied to a crack of length $2 a$ in a large elastic body. To illustrate the size dependency of $|\mathrm{d} W / \mathrm{d} A|$ on an element ahead of the crack, an estimate will be made for silica glass fibers $\left(\approx 10^{-4} \mathrm{~cm}\right.$ diameter $)$ that were studied in [9]. Their mechanical properties are given in [7] referring to Fig. 8(a) for $\theta=0$, i.e., an element directly ahead of the macrocrack. Eq. (16) gives the volume energy density

$\frac{\mathrm{d} W}{\mathrm{~d} V}=\frac{(1+v)(1-2 v)}{2 E_{0}} \sigma^{2}\left(\frac{a}{r}\right)$,

based on the asymptotic solution which is the exact solution for $a / r=10$. The remote stress $\sigma$ is related to the local stress pulling on the element with an ultimate strength $\sigma_{\mathrm{u}}$ for silica glass, i.e.,

$\sigma^{2}=\frac{1}{4}\left(\frac{\rho}{a}\right) \sigma_{\mathrm{u}}^{2}$

The surface flaws are assumed to be $10^{-3} \mathrm{~cm}$ in length and their sharpness is specified by $\rho / a=10^{-1}$.

For silica glass, $E_{0} / \sigma_{\mathrm{u}}=7.35, v=0.25, \sigma_{\mathrm{u}}=10$ GPa, Eq. (18) can be substituted into Eq. (27) to give $\mathrm{d} W / \mathrm{d} V=10.629 \times 10^{7} \mathrm{~Pa}$. The stresses in Eqs. (15) for plane strain and $\theta=0^{\circ}$ are given by

$\sigma_{x}=\sigma_{y}=\frac{\sigma \sqrt{a}}{\sqrt{2 r}}, \quad \sigma_{z}=\frac{v \sigma \sqrt{2 a}}{\sqrt{r}}$,

$\tau_{x y}=\tau_{y z}=\tau_{x z}=0$.
The corresponding non-vanishing strains are

$\varepsilon_{x}=\varepsilon_{y}=\frac{(1+v)(1-2 v)}{E_{0}} \frac{\sigma \sqrt{a}}{\sqrt{2 r}}$.

Substituting Eq. (30) into Eqs. (24), it can be easily deduced from Eq. (25) that $|\mathrm{d} V / \mathrm{d} A|=3 \ell_{0}$, where $\ell_{0}$ is taken as $10^{-6} \mathrm{~m}$. These results can be substituted into Eq. (26) to yield $|\mathrm{d} W / \mathrm{d} A|=$ $318.87 \mathrm{~Pa} \mathrm{~m}$. It applies to the energy transmitted into three orthogonal surface area of an element ahead of a crack.

Note that $\mathrm{d} W / \mathrm{d} A$ and $\mathrm{d} W / \mathrm{d} V$ must be mutually interactive because surface and volume coexist as part of the same body. Under rising load, material discontinuities grow gradually in size according to the degree of homogeneity. For definiteness sake, consider element sizes of $10^{-4}, 10^{-3}$, and $10^{-2} \mathrm{~cm}$ in lineal dimension. While energy is transmitted across a surface (or interface), the surface energy density would increase with time such that small size discontinuity occurs earlier at some critical value $(\mathrm{d} W / \mathrm{d} V)_{\mathrm{c}}$. Larger size discontinuities would follow in time as load continues to increase, Fig. 12(a). The variations of volume energy density $\mathrm{d} W / \mathrm{d} V$ would follow an opposite trend, i.e., the curves would increase with time, slowly at first and then more rapidly, Fig. 12(b). These time characteristics of imperfection size behavior have been analyzed by the non-equilibrium thermomechanics theory $[3,4]$.

\subsection{Crystal size determination}

The nucleation process of crystals depends on a host of factors such as dislocations, pre-existing heterogeneities, etc. In the ideal case of a spherical nucleus of radius $R$, the Gibbs' free energy $\Delta G$ for the homogeneous nucleation is given by

$\Delta G=4 \pi R^{2} \Delta G_{\mathrm{s}}+\frac{4}{3} 4 \pi R^{3} \Delta G_{\mathrm{v}}$.

The critical radius $R_{0}$ can be found by taking $\mathrm{d}(\Delta G) / \mathrm{d} r=0$, i.e.,

$\frac{R_{0}}{2}=\frac{\Delta G_{\mathrm{s}}}{\Delta G_{\mathrm{v}}}$.

It follows that the critical activation energy for nucleation is $\Delta G_{\mathrm{c}}=(16 \pi / 3)\left(\Delta G_{\mathrm{s}}^{3} / \Delta G_{\mathrm{v}}^{2}\right)$. In fact, 


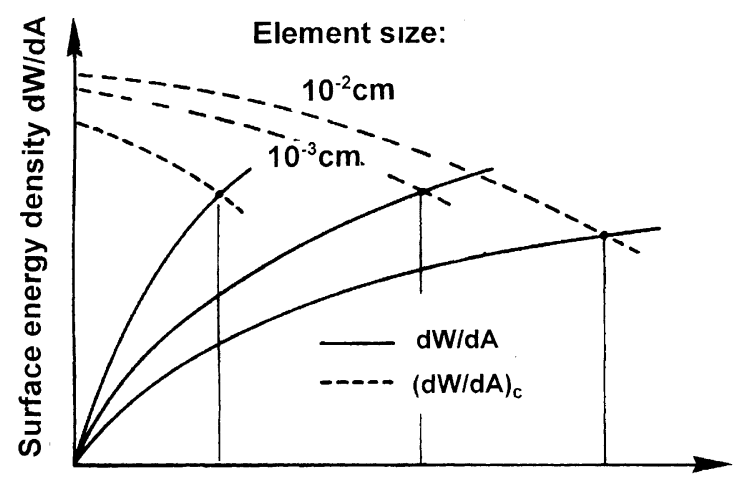

(a)

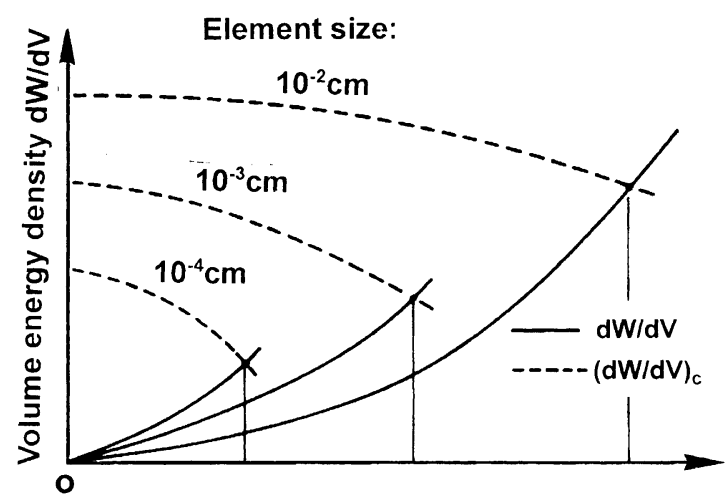

(b)

Time $\mathbf{t}$

Fig. 12. Size and time interaction of surface and volume energy density: (a) surface energy; (b) volume energy.

Eq. (32) can be derived directly from Eq. (23) or Eq. (26) where $\mathrm{d} V / \mathrm{d} A$ is a scalar for the uniform expansion of the spherical body. For $V=4 \pi R^{3} / 3$ and $A=4 \pi R^{2}, \mathrm{~d} V / \mathrm{d} A=R / 2$ and Eq. (32) is in fact Eq. (26).

The quantities $\mathrm{d} V / \mathrm{d} A$ must remain finite in the crystal nucleation process; they determine the mismatches from crystal to crystal at the interfaces as well as the sizes of crystals.

\section{Multiscale cracking behavior}

Interaction of macrocrack with microcracks involves materials that are damaged at different scale levels. This class of problems [29-34] may require two orders of magnitude sensitivity to capture the detailed features of the macro- and microcrack interaction if they were to be solved in a single simulation. An alternative would be to solve macro- and microcrack problems separately. The results are then connected by using a scaleindependent criterion based on "force" or "energy". Scale shifting factors can thus be derived to transfer stress defined at one scale level to another, say from macro- to micro- or mesoscale.

\subsection{Scale of material structure and imperfection}

Modeling multiscale features involves a description of the material structures and the corresponding physical imperfections that possess patterns and size scales of their own. While no intention is made to develop the formalism and convention for their classification, it is necessary to distinguish the different features at the different scales as a matter of convenience for reference. What the naked eye could see serves as a logical base of reference (macroscopic) from which the scale in lineal dimension can spread out in ascending and descending order of size. For the lack of a better choice, this work shall adopt the following convention on the centimeter length scale:

Macro: $10^{-3}-10^{-2} \mathrm{~cm}$,

Meso: $10^{-5}-10^{-3} \mathrm{~cm}$,

Macro: $10^{-6}-10^{-5} \mathrm{~cm}$.

The mesoscale not referred to ordinarily is added to refine divisions in lineal dimension between the micro- and macroscale; it still covers two orders of magnitude in $\mathrm{cm}$ and may require further refinement.

Geometric features of material structure involve atoms, arrangement of atoms in crystal lattice, grain, grain cluster, etc. Imperfections are created at each scale level according to the geometry and energy state of the material structure. They may include dislocations, voids, inclusions, microcracks, etc. The picture Fig. 13(a) shows a BCC crystal lattice $\left(10^{-8} \mathrm{~cm}\right)$. Imperfections at this scale could appear as dislocations in a grain as slip line or at coherent grain boundaries as edge dislocations. Voids and inclusions $\left(10^{-6}-10^{-5} \mathrm{~cm}\right)$ are 


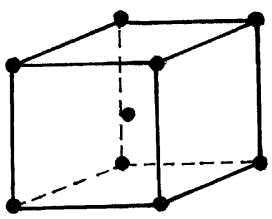

(a)

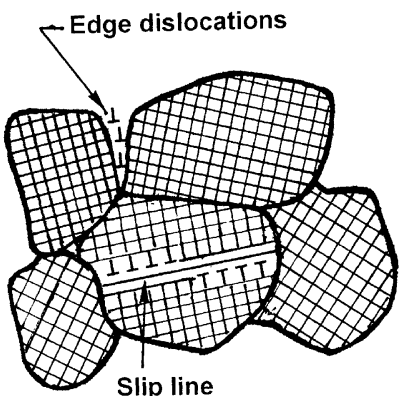

Slip line

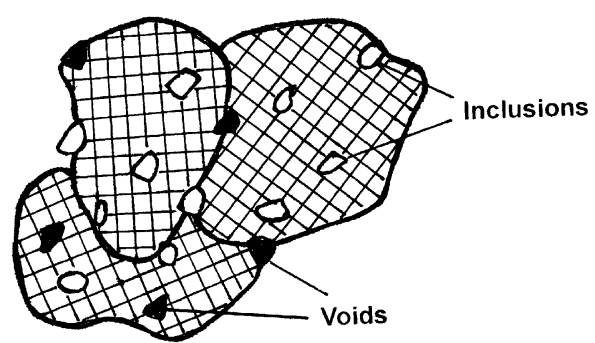

(b)

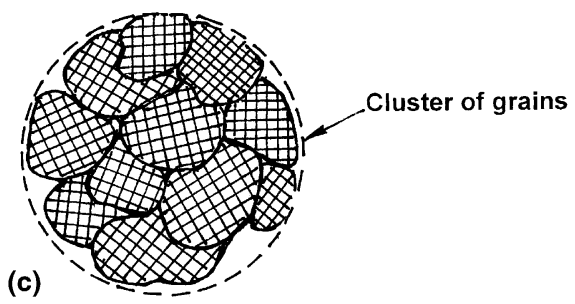

Fig. 13. Detail features of material microstructure and associated imperfections: (a) lattice and dislocation $\left(10^{-8} \mathrm{~cm}\right)$; (b) voids and inclusions $\left(10^{-6}-10^{-5} \mathrm{~cm}\right)$; (c) grains $\left(10^{-5}-10^{-3} \mathrm{~cm}\right)$.

often found in the grain and at the grain boundaries, Fig. 13(b). Note that grains can also react as a cluster $\left(10^{-5}-10^{-3} \mathrm{~cm}\right)$. This is shown in Fig. 13(c). Details of the atomic level $\left(10^{-8} \mathrm{~cm}\right)$ would entail electrons spinning about the nucleus $\left(10^{-13} \mathrm{~cm}\right)$ that contains the protons and neutrons. Nanoscale technology has already merged into the region of electron cloud. At this scale level, imperfections are no longer associated with geometry but with inhomogeneities in the energy density field of electrons. An interface [35,36] would be associated with the barrier where the densities of the electrons on both sides are different. It is not surprising that the particle physicists have long recognized the association of size with energy level, i.e., the equivalence of $\mathrm{a} \mathrm{GeV}$ to distance of $10^{-4} \mathrm{~cm}$.

\subsection{Scaling in mechanics}

Classical continuum mechanics is said to have left out the size effect when letting the element size or $\mathrm{d} V / \mathrm{d} A$ to approach zero in the limit. The difference between letting a mathematical quantity to approach zero and having it to be identically zero may appear to be subtle. It has far-reaching im- plications. This can be illustrated using the theory of elasticity as an example. Recall that the classical boundary traction conditions in Eq. (1) are obtained from Eq. (2) by letting $\mathrm{d} V / \mathrm{d} A$ to vanish in the limit as the continuum element shrinks to zero. It is clear that the increment change in volume $\Delta V$ of an element in elasticity is the first strain invariant given by

$\Delta V=\varepsilon_{x}+\varepsilon_{y}+\varepsilon_{z}$,

as discussed earlier in relation to Eq. (27) or Eq. (26).

Clearly, $\Delta V / \Delta A$ (or $\mathrm{d} V / \mathrm{d} A$ ) is not zero even though the limit $\Delta V / \Delta A \rightarrow 0$ was invoked in the formulation of the theory. The implication is that $\Delta V / \Delta A$ from the $j$ th element to the nearest $(j+1)$ th element should be confined within a limit

$\left|\left(\frac{\Delta V}{\Delta A}\right)_{j}-\left(\frac{\Delta V}{\Delta A}\right)_{j+1}\right|=\delta \ll$.

This limit $\delta$ should be as small as possible. Eq. (33) implies a length scale limitation in continuum mechanics theories, the implications of which associated with finite element numerical calculation can be found in [37]. 


\subsection{State of affairs near crack tip}

The free surface creation model in [9] was applied to analyze cracking of solids for more than two decades before attempts were made to associate it with the crack tip detail features. X-ray photographic results of apparently brittle fracture of low carbon steel [38] were reported to have detected a thin layer of material at the surface that contains significant plastic deformation. A quantity $\gamma_{p}$ referred to as "plastic work" was introduced and estimated to be of the order of $10^{3}$ times greater than $\gamma_{\mathrm{s}}$ surface tension measured in [9]. The critical macrostress relation was modified as

$\sigma \approx \sqrt{\frac{2 E_{0}}{\pi a}\left(\gamma_{\mathrm{s}}+\gamma_{\mathrm{p}}\right)}=\sqrt{\frac{2 E_{0}}{\pi a} \gamma_{\mathrm{p}}}$.

It was argued [38] that since $\gamma_{\mathrm{p}} \gg \gamma_{\mathrm{s}}$ the original expression of $\sigma$ [9] for plane stress could be modified simply by letting $\gamma_{\mathrm{s}}$ equal to $\gamma_{\mathrm{p}}$. Such an interpretation, however, could not be justified by comparing physical quantities at different scale levels. This was pointed out in [8] that a scale shifting factor should be used when relating scaledependent quantities from one scale to another. A factor in the term $\sqrt{2 a_{0} E_{0} / \pi \rho e_{0}}$ was suggested such that Eq. (34) should have been written as

$\sigma_{\text {macro }}=\sqrt{\frac{2 E_{0}}{\pi a}} \sqrt{\frac{2}{\pi}\left(\frac{a_{0}}{\rho}\right)\left(\frac{E_{0}}{e_{0}}\right)} \sqrt{\gamma_{\text {micro }}}$.

The middle term on the right-hand side of Eq. (37) was referred to [8] as the "scale shifting factor". If $a_{0}$ and $e_{0}$ are taken as microscopical parameters, then $\gamma$ in Eq. (37) will follow such that $\gamma \rightarrow \gamma_{\text {micro }}$. All quantities on the right of Eq. (34) without subscripts refer to the macroscopic scale. More precisely, Eq. (37) was derived from the relation

$\frac{\gamma_{\text {micro }}}{\gamma_{\text {macro }}}=\frac{\pi}{2}\left(\frac{a_{0}}{\rho}\right)\left(\frac{e_{0}}{E_{0}}\right) \approx 10^{3}-10^{4}$,

which can be applied between any two different scale levels.

A descriptive account of the state of affairs near a Mode I crack tip can be found in Fig. 14. Directly ahead of the crack, the macroelement $\Delta x \Delta y$ is in a state of hydrostatic tension when dilatation would dominate. This can be shown from the re- sults in Eqs. (18) and (19). However, the smaller distortion energy density $(\mathrm{d} W / \mathrm{d} V)_{\mathrm{d}}$ could contribute to shear of a thin layer at a lower scale level which could have been the layer referred to in [38]. By the same token the element off to the side of macrocrack is under severe distortion as described by Eqs. (20) and (21). The dilatational energy density $(\mathrm{d} W / \mathrm{d} V)_{\mathrm{v}}$ in Eq. (20) is smaller than $(\mathrm{d} W / \mathrm{d} V)_{\mathrm{d}}$ but not negligible; it yields a dilatational field at a lower scale level and could lead to small cracks in a slanted zone, Fig. 14. Such lower scale cracks normal to the load have been reported by experiments. These smaller cracks have been loosely referred to in the past as microcracks. More exact measurements should be made to record their sizes in order to better understand the mechanisms associated with their creation for a particular material structure and strain rate.

\subsection{Scaling range}

One of the key issues in fracture mechanics has been involved with establishing greater precision in the analytical description of multiscale fracture/ failure problems. This would require a knowledge of the range of characteristic lengths that could be embedded in a single simulation. Is it realistic to perform calculations covering both the macroscopic and microscopic effects or must it be done separately and then seek for conditions to connect the results? Keep in mind that almost all presentday calculations are made by invoking equilibrium mechanics assumptions. That is for a closed thermodynamic system where boundary conditions are specified rather than treated as unknowns for an open thermodynamic system $[3,4]$. It is still puzzling as to how the scale-discrete solutions could be connected without knowing that some pertinent information could have been smeared at the termination of one scale and the start of another. To put it more bluntly, the connection of macroscopic and microscopic results as stated may not even be a valid choice. There is the problem of synchronizing the size, time and temperature variables at the same scale level $[3,4]$ not to mention how information could be uniquely transferred from one scale to another. In principle, a one-to-one correspondence should be observed between the results 


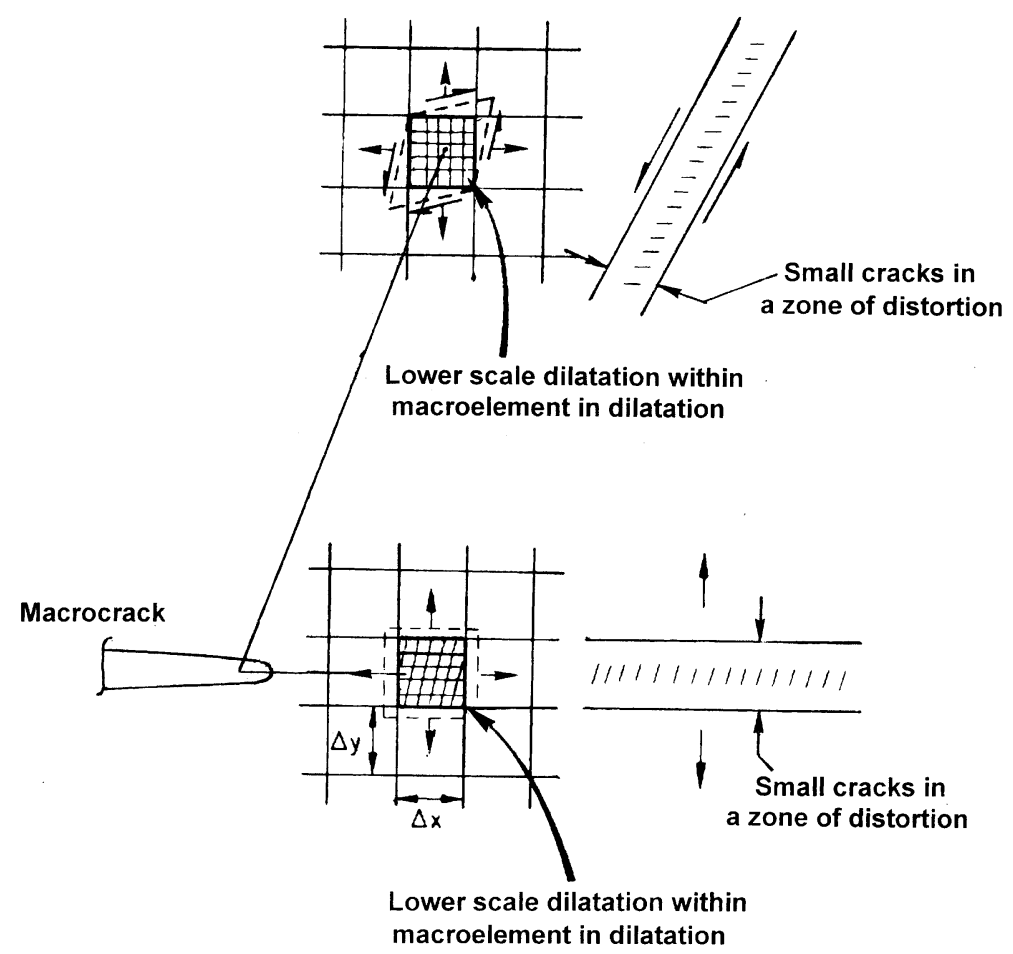

Fig. 14. Zones of distortion and dilatation ahead of a macrocrack in tension.

for two adjoining scales, the range of which should be established by rational means rather than intuition. Generally speaking, a fixed set of microscopic entities would yield a unique macroscopic average. The converse may not hold. That is a macroscopic average may correspond to several smaller scale detailed features. Scale interactions do not reciprocate. It is, therefore, useful to establish cross-scale criteria based on "force" or "energy" that are independent of scale. It would be incorrect to equate microstress to macrostress or microstrain to macrostrain for their definitions are size dependent. Macrostress and microstress refer to respective units of $\mathrm{MPa}$ and $\mathrm{GPa}$; they address defects that may have several orders of magnitude difference.

The relation given in Eq. (38) for two different scale levels may be established by using the force criterion. It could not distinguish macrofracture from microfracture or vice versa. Suppose that incipient fracture is assumed to occur at a critical force $f_{\mathrm{c}}$ which can be written in terms of stresses as $\sigma_{\text {micro }} \cdot \Delta L_{0} \cdot 1=\sigma_{\text {macro }} \cdot \Delta L \cdot 1$,

where $\Delta L_{0}$ and $\Delta L$ refer, respectively, to microscopic and macroscopic element sizes with unity in thickness for a two-dimensional model. The same applies to the energy criterion

$2 \gamma_{\text {micro }} \cdot \Delta L_{0} \cdot 1=2 \gamma_{\text {macro }} \cdot \Delta L \cdot 1$.

The factor 2 is indicative of the creation of the upper and lower crack segments. Eq. (40) gives

$\frac{\gamma_{\text {micro }}}{\gamma_{\text {macro }}}=\frac{\Delta L}{\Delta L_{0}}=10^{3}-10^{4}$,

which is an inverse length proportional relation for scale shifting. Eqs. (39) and (40) show that the force and energy criterion are equivalent:

$\frac{\sigma_{\text {micro }}}{\sigma_{\text {macro }}}=\frac{\gamma_{\text {micro }}}{\gamma_{\text {macro }}}$.

Making use of Eq. (5) for $4 e_{0}=E$ with $\sigma_{\mathrm{c}}$ and $\gamma_{\mathrm{c}}$ being microscopic and using the original equation $\sigma=\sqrt{\left(2 E_{0} / \pi a\right) \cdot \gamma_{\mathrm{s}}}$ [9] with $\gamma_{\mathrm{s}}$ and $\sigma$ being mac- 
roscopic, there results Eq. (38). As mentioned earlier, the form of Eq. (38) holds for any two scale levels where the stress-strain relationships were assumed to be linear. Correction for non-linearity is not the essence of this discussion. What is more relevant is to establish the order of magnitude of Eq. (41) and the corresponding physical mechanisms associated with the surface creation process, say $\gamma_{\text {nano }}, \gamma_{\text {micro }}, \gamma_{\text {meso }}$, and $\gamma_{\text {macro }}$. The gap between $\gamma_{\text {micro }}$ and $\gamma_{\text {macro }}$ as stated in Eq. (41) should perhaps be shortened to that of $\gamma_{\text {macro }}$ and $\gamma_{\text {meso }}$. Based on the experimental observations in $[13,14]$, it might be worthwhile to examine whether indeed the creation of mesocracks is intimately associated with the action of shear + rotation.

\subsection{Stable and non-stable growth}

The current trend of miniaturization has called for greater details of the material structure. Reduction in size appears to be traded by an increase in speed $[39,40]$. Temperature also interacts and tends to decrease. These effects can be predicted in a consistent fashion using the non-equilibrium thermomechanics theory developed in $[3,4]$. Atomic simulations could not resolve the fundamental problem of scale interaction because the solution depends on the boundary conditions at the atomic scale level. They are in fact unknowns and should not be assumed intuitively or otherwise.

In view of the lack of consistency for specifying the spacial/time/temperature interactive effects, interpretation of test results would be premature until the physical parameters are shown to remain valid within the scale range they are defined. Macroscopical definitions rely on material homogeneity; they cannot be applied to explain local events that are dictated by microscopic detail features. Mixing scale sensitive quantities introduces arbitrariness and uncertainties into the outcome.

Recent works [41-43] on cracks that undergo subsonic-to-intersonic transition should be scrutinized in view of the foregoing comments. To reiterate, "transition" represents a break-down or range limitation of a mathematical formulation. The piecemeal treatments of subsonic, transonic and supersonic problems simply mean that these phenomena have to be analyzed in segments. No such distinctions need to be made in the thermomechanics theory $[3,4]$. To claim that a crack has propagated beyond the Rayleigh wave speed as a contraction in continuum mechanics [43] would be unjustified should the explanation lie simply in a violation of the definition of Rayleigh wave speed. It is a continuum mechanics parameter that applies strictly to a homogeneous situation.

On physical grounds, the speed of a crack propagating in a self-similar manner could increase or decrease depending on the resistance offered by the material ahead. Suppose that the crack tip radius of curvature and the material weakens in time, then the crack speed would increase monotonically for the same driving force or available energy. There is no reason why the speed of a crack with a smaller opening would not exceed that with a wider opening.

Interaction of macro- and microcracks is also common in brittle composites and ceramics. Damage may not always be localized to the immediate vicinity of a dominant crack front. Test results for ferroelectric ceramics [44] have shown that the cyclic load data deviated from the correlation for the growth of a single dominant crack when microcracking increased. An additional parameter was thus needed [33,34] to measure the extent of microcracking in relation to the critical ligament that would trigger the onset of macrocrack instability. The model would thus account for cracking at both the macro- and micro- or mesoscale levels.

Microcracking dissipates energy that would have otherwise been present to advance the macrocrack. Let damage by microcracking be reflected on the average by the length $r_{\mathrm{f}}$ while $r_{\mathrm{c}}$ is the ligament that triggers the onset of rapid macrocrack growth. The ratio

$\lambda=\frac{r_{\mathrm{f}}}{r_{\mathrm{c}}}, \quad r_{\mathrm{f}}<r_{\mathrm{c}}$

measures the extent of stable microcrack growth as compared to $r_{\mathrm{c}}$. The volume energy density criterion [16-18] distinguishes stable slow crack growth from unstable fast crack propagation. The former 
condition corresponds to $\mathrm{d} W / \mathrm{d} V$ reaching a critical value

$$
\left(\frac{\mathrm{d} W}{\mathrm{~d} V}\right)_{\mathrm{c}}=\frac{S_{\mathrm{f}}}{r_{\mathrm{f}}} \rightarrow \frac{S_{\mathrm{c}}}{r_{\mathrm{c}}} \quad \text { (unstable fracture) }
$$

In Eq. (44), $S_{\mathrm{f}}$ and $r_{\mathrm{f}}$ are parameters that reflect damage by stable microcracking. Unstable macrofracture is assumed to occur when $S_{\mathrm{f}} / r_{\mathrm{f}} \rightarrow S_{\mathrm{c}} / r_{\mathrm{c}}$. For the group of PZT ferroelectric ceramics subjected to mechanical stress $\sigma$ and electric field $E$, the energy density factor $S$ is given by [34]

$S=B_{11} K_{1}^{2}+2 B_{14} K_{1} K_{E}+B_{44} K_{E}^{2}$,

in which

$K_{1}=\sigma \sqrt{\pi a}, \quad K_{E}=E \sqrt{\pi a}$.

The constants $B_{11}, B_{14}$ and $B_{44}$ are complicated functions related to the elastic, piezoelectric and dielectric constants of the PZT ferroelectric ceramics. Eqs. (46) can be inserted into Eq. (45) to give

$S=K_{1}^{2}\left[B_{11}+2 B_{14} p+B_{44} p^{2}\right]$,

where $p=E / \sigma$ is a load parameter.

The threshold conditions in Eqs. (44) can be established experimentally by referring to mechanical loads only such that

$S_{\mathrm{f}}=B_{11} \sigma_{\mathrm{f}} \sqrt{\pi a}, \quad S_{\mathrm{c}}=B_{11} K_{\mathrm{IC}}^{2}$.

Making use of Eqs. (43), (44), (47) and (48), an expression for failure stress is obtained:

$\sigma_{\mathrm{f}}=\frac{K_{\mathrm{IC}}}{\sqrt{\pi a}} \sqrt{\frac{\lambda}{F(p)}}$.

In Eq. (49), the function $F(p)$ stands for

$F(p)=1+2\left(B_{14} / B_{11}\right) p+\left(B_{44} / B_{11}\right) p^{2}$.

For $\lambda=1$, crack initiation corresponds to the onset of rapid fracture, i.e., when $\sigma_{\mathrm{f}}$ becomes the critical stress to trigger macroscopic instability. Hence, the stress to initiate microcracking as given by Eq. (49) will also precede that to drive the macrocrack.

The energy release rate concept accounts only for the onset of free surface creation; it does not address stable from unstable crack growth. It is even less remotely concerned with brittle and ductile fracture [38]. Unsuccessful attempts made to explain the enhancement and retardation of crack growth due to electric field reversal [31,32] are indicative of the limitation of the energy release rate concept.

\section{Implication of asymptotic and full field solutions}

Analytical modeling of physical events is not always a unique process. Agreement between theory and experiment is necessary but may not be sufficient for validating a given approach, particularly when the predictions from different methods are all within experimental errors. Compensating discrepancies could also arise from analyses and/or experimental measurements. It is, therefore, prudent not to overlook any potential pitfalls even when the results might appear to be unfamiliar at first sight.

A resurgence of interest has recently been centered on crack bifurcation for a stationary [49] and moving [46] crack where the investigations involve events at the micro-, meso- or macroscopic scale. Whether continuum mechanics theories could still be applied at these size scales deserves attention.

\subsection{Application of continuum mechanics}

Continuum mechanics theories are not concerned with size effects and hence they are not pinned down to any particularly size scale. The general notion that elasticity is limited to macroscopic behavior is simply because the prevailing elastic constants are determined according to the ASTM standards and describe the bulk properties of specimens of the order of $10 \mathrm{~cm}$ in lineal dimension. There are no a priori reasons why elasticity could not be applied to the atomic scale if the electron structure of the material is accounted for determining the physical constants. This indeed is the traditional practice in crystal physics. Recent studies [29-34] dealing with the application of piezoelasticity to model multiscale damage of PZT ceramics have encountered many physical events that are not well understood. Some of the areas identified for discussion are: 
- Interaction of the tetragonal perovskite structure of PZT ceramics with the direction of applied electric field relative to poling and its effect on crack growth [29-34].

- Distance and orientation dependence of crack initiation sites in piezoelectric materials associated with multiscale damage.

- Exclusion of multiscale behavior in analyses due to invoking of the limiting process inherent in the assumed fracture criterion.

- Insufficient understanding of approximation associated with the asymptotic solution for a crack-like discontinuity in contrast to the full field solution.

The foregoing areas of concern are interconnected even though they could be addressed individually. Within the framework of piezoelasticity, the energy density fracture criterion will be used for discussion since it has already been introduced [17] in this work.

\subsection{Asymptotic approximation}

As the crack tip is approached or is in the limit as $r \rightarrow 0$ in Eq. (8), only the singular term in the volume energy density function $\mathrm{d} W / \mathrm{d} V$ remains. The coefficient $S$ becomes independent of $r$; it varies with the angle $\theta$ only which is measured from a line extending from the crack front. The quotient $S / r$ in Eq. (8) is referred to as the asymptotic crack tip solution. No reference is made to a particular location ahead of the crack except that it applies to the immediate vicinity of the crack tip.

To be specific, reference shall be made to the problem presented in $[33,34]$ that deals with a finite crack of length $2 a$ in a piezoelectric material (PZT4) that is infinite in extent (plane strain). A uniform electric field $E$ and uniaxial tensile stress $\sigma$ are applied at infinity. Poling is directed in the positive $y$-direction while the crack plane coincides with the $x$-axis. The corresponding energy density factor $S$ is given by Eq. (47) in which $p$ stands for the ratio of the applied electric field $E$ and the applied stress $\sigma$, i.e., $p=E / \sigma$. The quantities $B_{11}$, $B_{14}$ and $B_{44}$ are complicated constants for the PZT4 material. Details can be found in $[33,34]$.
The procedure to obtain the direction of crack initiation involves finding the stationary values of $S$ with respect to $\theta$, where $r$ is fixed in the asymptotic field solution. Many minima of $S$ could be found. However, it is the maximum of $S_{\min }$ that would first reach critical values. Mathematically, it can be stated as $S_{\min }^{\max } \rightarrow S_{\mathrm{c}}$. For illustration, it suffices to single out three typical cases, where $p=E / \sigma$ is allowed to vary while the other parameters are fixed.

- Case I ( $S / r$-asymp). Stationary values or $S_{\min }$ do not exist for $p=-0.03$ and $-\pi<\theta<\pi$.

- Case II ( $S / r$-asymp). For $p=-0.05$, no $S_{\min }$ values are found for $\theta=0^{\circ}$ but they do exist for $\theta= \pm \theta_{0}$ corresponding to the angles of bifurcation.

- Case III ( $S / r$-asymp). For positive $p, S_{\min }$ values exist for $\theta=0^{\circ}$.

The above findings are summarized graphically in Fig. 15. They show that the existence of $S_{\text {min }}$ depends on whether $p$ is positive or negative. There is a narrow range of negative $p$ values within which $S_{\min }$ do not exist. This peculiar behavior occurs only in piezoelasticity and not in elasticity crack problems, at least at size scale level that is being analyzed. There is no apparent reason why Mode I crack initiation for $\theta=0^{\circ}$ is not predicted for negative values of $p$ in the range where 0.03 and 0.05 prevail. Crack bifurcation $\theta= \pm \theta_{0}$, however, is predicted in Case II for $p=-0.05$ but not for $p=-0.03$. Such a behavior is also unexpected for a stationary crack as bifurcation is generally identified with the crack approaching its terminal

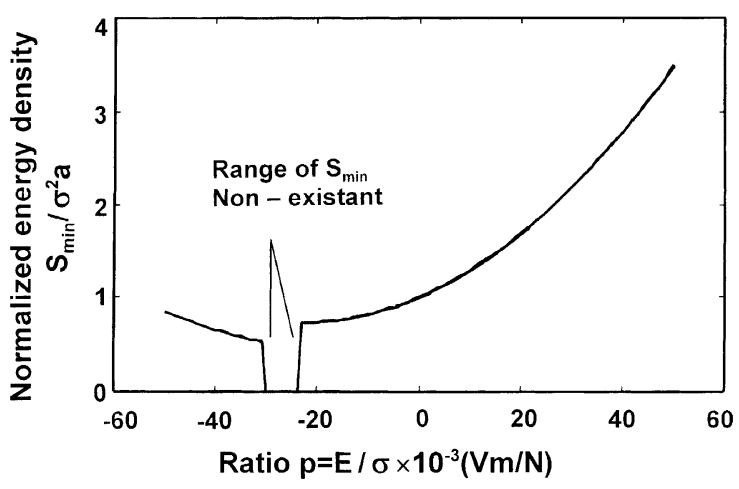

Fig. 15. Discontinuity in normalized energy density curve. 
velocity [50]. When $p$ becomes positive, say 0.02 and larger, $S_{\min }$ would always exist for $\theta=0^{\circ}$, Fig. 15. That is crack initiation along the $x$-axis is predicted.

Even though continuum mechanics theories can be applied to any scale, the use of asymptotic field solution could not guarantee the inclusion of details in a region local to the crack tip. This is reminiscent of the unexpected "wiggling" of crack path emanating from a notch [51] that would not have been predicted if the full field solution of $\mathrm{d} W / \mathrm{d} V$ were not used. The asymptotic solution would have missed out all of the crucial details.

\subsection{Full field solution}

Since the energy density criterion as stated in [17] applied to all continuum mechanics solutions whether in asymptotic or complete form, the criterion can be applied to analyze $\mathrm{d} W / \mathrm{d} V$ computed numerically. The three cases presented earlier for $S / r$-asymp will be used to obtain $\mathrm{d} W / \mathrm{d} V$-full for comparison. The additional consideration would involve $r / a$ which did not enter into the discussion for $S$ being independent of $r$.

- Case I (dW/dV-full). For $p=-0.03$ and $r / a>10^{-3}$, stationary values $(\mathrm{d} W / \mathrm{d} V)_{\min }$ are found to exist for $\theta=0^{\circ}$.

- Case II (d $W / \mathrm{d} V$-full). When $p=-0.05$, $(\mathrm{d} W / \mathrm{d} V)_{\min }$ exists for $\theta=0^{\circ}$ and $\theta= \pm \theta_{0}$ depending on the relative distance $r / a$. More precisely, crack bifurcation $= \pm \theta_{0}$ is predicted for $r / a<10^{-2}$ and crack extending straight ahead $\theta=0^{\circ}$ is predicted for $r / a>10^{-2}$.
- Case III ( $\mathrm{d} W / \mathrm{d} V$-full). For positive $p$, $(\mathrm{d} W / \mathrm{d} V)_{\min }$ exists only for $\theta=0^{\circ}$.

In contact to the $S_{\min }$ predictions, predictions based on the full field solutions of $(\mathrm{d} W / \mathrm{d} V)_{\min }$ are all physically plausible. For multiscale problems, $(\mathrm{d} W / \mathrm{d} V)_{\min }$ depends on the relative distance $r / a$. Straight ahead (or Mode I) crack extension is predicted for $p=-0.03$ only if $r / a>10^{-3}$, i.e., not at the immediate vicinity of the crack tip. This is consistent with Case I ( $S / r$-asymp) where $S_{\min }$ was not predicted. It is conceivable that very close to the crack tip, say at the atomic or molecular scale, distortion would dominate due to the lack of material stiffness or dilatation and hence the emission of dislocations in the form of bifurcation is known to prevail. Even more revealing are the predictions of Case II ( $\mathrm{d} W / \mathrm{d} V$-full) for $p=-0.05$ where bifurcation $\left(\theta= \pm \theta_{0}\right)$ was predicted to be very close to the crack tip while straight ahead cracking $\left(\theta=0^{\circ}\right)$ was predicted at a distance away from the crack tip where dilatation becomes more dominant. Such a multiscale behavior is shown schematically in Fig. 16. Note that the bifurcation region $r<10^{-2} a$ could represent dislocation emissions. For $a \approx 1 \mathrm{~cm}$, presumably $10^{4}$ or more dislocations could be piled into a local region on a plane. Straight ahead macrocracking would be predicted outside of this microscopic region. The dual scale behavior has apparently been exhibited in a single simulation by application of the full field solution. According to this example for piezoelasticity, the multiscaling fracture was found to be most pronounced in the range where $S_{\min }$ did not appear at the scale level under consideration.

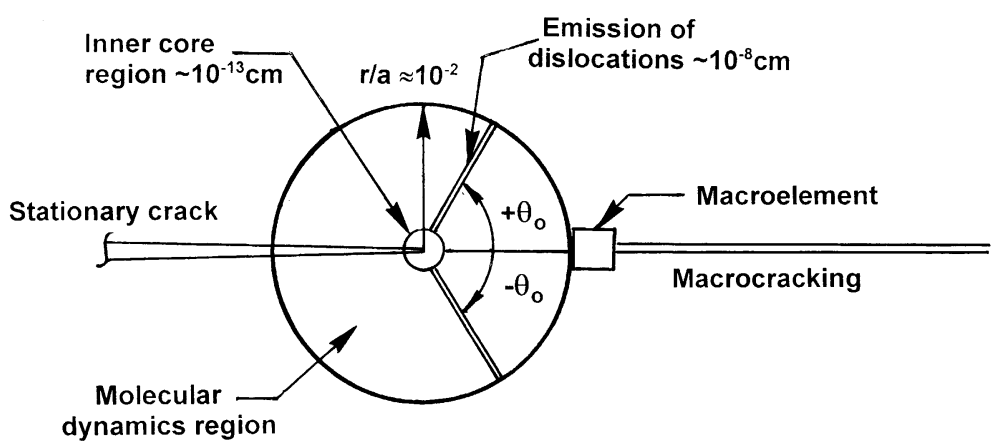

Fig. 16. Multiscaling behavior ahead of stationary crack for PZT-4 and $p=-0.05$. 
As $p$ becomes positive, only the straight ahead cracking behavior is predicted by both the asymptotic and full field solution. They correspond to Case III $(S / r$-asymp) and Case III $(\mathrm{d} W / \mathrm{d} V$ full).

The implication is that there might exist a transition region in which the bifurcation angles $\pm \theta_{0}$ would disappear as $r / a$ is increased and only one branch would prevail. According to the $\mathrm{d} W / \mathrm{d} V$-criterion, distortion could decrease with increasing $r / a$ in this scale range. Once sufficient dilatation is built up, a single branch could dissipate the energy. What is being altered during this process is the rate of energy dissipation which is higher for dilatation. The distortional/dilatational/ distortional... flip-flop mechanism of energy dissipation tends to follow the ascending (or descending) size scale. It has been elaborated in [15] and earlier works. These aspects of multiscale damage can now be illustrated quantitatively by using the example for the PZT-4 ceramic. More on this will follow. Hence, asymptotic and full field solutions do not always yield the same predictions even though the same fracture criterion were used. In the case of piezoelasticity, the result depends on the ratio of $E / \sigma$ and the direction of the applied electric field relative to poling. That is the microstructure of the piezoelectric material had an effect on the ways with which damage occurred ahead of a crack.

To reiterate, bifurcation could occur for a stationary crack provided that it takes place very close to the crack tip where dislocations could be emitted as shown in Fig. 16. The precise mechanism of material damage due to slip or microsurface separation remains to be examined. Atomistic and micromechanics models $[47,48]$ do assume that the emitted dislocations are confined within the molecular dynamics region; they are assumed not to cross the micro-macrobarrier $\Gamma$, Fig. 16. This artificial adjustment has been $[47,48]$ made as an a priori in the analyses, not predicted. The argument is that within $\Gamma$, the behavior is linearly elastic. What is surprising is that the energy density criterion is able to predict this dual scale feature of micro-macrofracture that would be normally pre-programmed into the atomistic/micromechanics model. It does appear that the molecular dynamics region in Fig. 16 should be better understood in relation to the transition details across the boundary $\Gamma$. The preliminary studies in [49] show that this dual scale behavior is sensitive to changes in the electromechanical loading and distances to the crack tip.

\subsection{Discrepancy estimate}

Discrepancies between the asymptotic and full field solution have been estimated [49]. They are not insignificant and depend on the external and internal field variables, some of which have already been mentioned. Two types of situations could arise. The asymptotic and full solution curves could intersect or be in parallel. The former
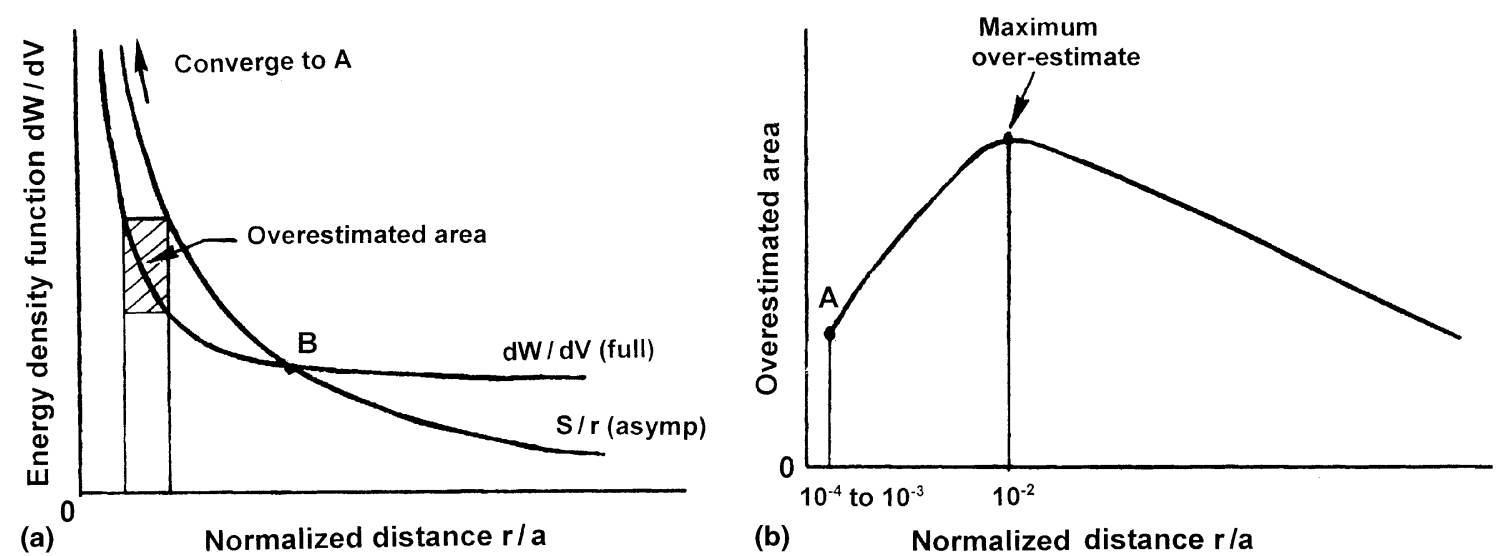

Fig. 17. Discrepancy between asymptotic and full field solution: (a) intersecting curves; (b) non-monotonic behavior. 
and latter represent an overestimate and underestimate of the asymptotic solution, respectively. The former case illustrated in Figs. 17(a) and (b) will be examined. Displayed in Fig. 17(a) are the curves that tend to meet at $A$ as $r / a \rightarrow 0$. Away from the crack tip, they would intersect at $B$. The overestimate is represented by the shaded area which can be computed as a function of $r / a$. This gives a non-monotonic curve that possesses a maximum at $r / a \approx 10^{-2}$ for the present problem. The point $A$ is located at $r / a \approx 10^{-4}$ and $10^{-3}$, Fig. 17(b); it can vary from problem to problem. At $r / a \approx 10^{-2}$, the asymptotic solution deviates the most from the full field solution.

While the foregoing conclusions tend to support the use of the full field solution numerically, simplicity of the asymptotic solution should not be overlooked. It can provide physical insights that otherwise could be hidden in the numerical data. A case in point is the form of Eq. (47) that has led to a quantitative assessment of the enhancement and impediment of crack growth in piezoceramics when the electric field direction is reversed relative to the poling direction [33,34].

\section{Non-linearity of dual scaling}

When plastic enclaves appear next to the sides of a macrocrack, it is common knowledge that the overall response would be non-linear. By the same token, linearity in the molecular dynamics region $[47,48]$ was assumed even though dislocations are known to be the source of plasticity. There is really no ambiguity if non-linearity is interpreted to be the cause of inhomogeneity arising from damage at two different scales. Clearly, a material in a state of uniform plasticity would respond linearly.

A more complete though speculative description of non-linearity associated with dislocations, plasticity and cracking can be found in Fig. 18. In the absence of a single microplastic enclave predicted by the $\mathrm{d} W / \mathrm{d} V$ criterion, response in the molecular dynamics region would be linear. As the macroplastic (or mesoplastic) enclaves become vanishingly small, the region becomes completely elastic and the macrocrack (or mesocrack) would behave in a linear fashion. The direction(s) of $\mathrm{d} W / \mathrm{d} V$ minimum or $(\mathrm{d} W / \mathrm{d} V)_{\min }$ corresponds to dilatational damage at the scale level of observation while the direction(s) of $(\mathrm{d} W / \mathrm{d} V)_{\max }$ to distortional damage. At the same time scale, the mechanism of distortion would precede that of dilatation. This has been emphasized in $[17,18]$ and the references therein. Keep in mind that size and time scales are interwoven; they would shift in synchronization. The single plastic enclave behavior in the molecular dynamics region would not have been detected if it were not for application of the energy density criterion to examine

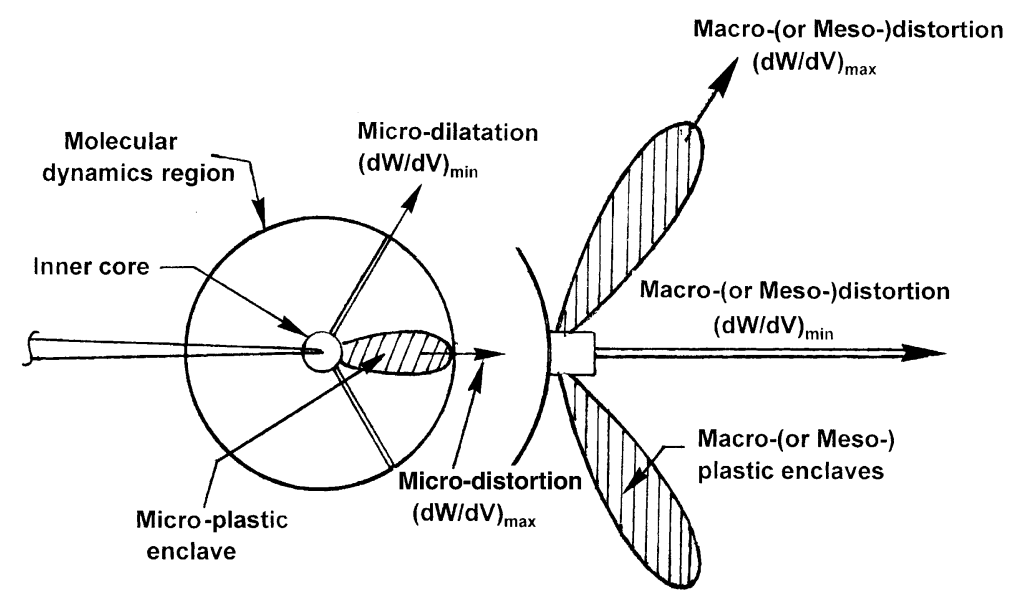

Fig. 18. Mechanisms of microdamage non-linearity and macrodamage (or mesodamage) non-linearity. 
cracking in PZT ceramics [49]. To reiterate, however, the speculative model in Fig. 18 is proposed to stimulate additional investigation.

\section{Concluding remarks}

Scaling is well known in aerodynamics where dimensionless groups have been established to transfer parameters measured during wind tunnel tests for the models to study the behavior of larger size structures. The same idea is invoked in structural mechanics of solids. Small specimen test data are collected, standardized and used in the design of full scale structures. Standardization is necessary for the sake of accumulating experience in practice. The objective of a theoretical analysis is not to predict or to replace tests rather it should be done to design an intelligent experiment. The intention is to correlate the data of two independent experiments: one being simpler and the other being more complex with the aim that tests could be simplified and the number of tests could be minimized. One of the main aims of fracture mechanics is to relate the uniaxial tensile test data to bodies containing one or more cracks. The challenge is to predict the fracture behavior of a cracked plate using uniaxial tensile data alone. Such a challenge was met in [45], where $K_{\mathrm{IC}}$ was determined analytically using a modified version of the theory of plasticity.

The process of scaling, however, cannot be reciprocated. That is to say the macroscopic average shed little or no light on the detail features of microscopic constituents. At present, the abundance of test data and analytical results are not being coordinated. There is the need to establish the formalism for a common set of terminologies such that the definition of physical quantities would not be violated when the scale levels of observation in size and time have exceeded certain limits. Special caution should be exercised when scale-sensitive quantities are exchanged from one scale to another.

Insufficient understanding of how to overcome some of the undesirable features of macrofracture behavior of brittle composites and structural ceramics has necessitated more detailed information of material structures at the atomic and microscopic levels. Graphical display of results obtained from atomistic calculations [46] suggests the presence of localized phonon-like modes near a moving crack tip, just prior to dislocation emission and crack branching events. Although these approaches provide additional motivation for research, equal amount of efforts should be placed on their contribution to improving the macroscopic properties of the material. Embedding a Molecular Dynamic computational model around the crack tip [47] and applying micromechanics to the surrounding region are an attempt to construct a two-scale model in a single simulation. Uncertainties at the boundary of the two regions, however, make the outcome somewhat artificial. It appears to be overly optimistic to bridge the gap between the atomic and microscopic scale and even more so for linking of the atomic and macroscopic views. The fundamental problem of how to specify the non-equilibrium boundary conditions at the atomic and microscopic scales requires attention.

Lacking in particular is a knowledge of the initial states of the material microstructure in terms of stress/strain or energy stored in the grain due to crystal nucleation and formation. The initial stresses and energies trapped in the material microstructure would no doubt have a first-order effect on the creation of dislocations, microvoids, grain boundary imperfections, etc. The neglect of these initial conditions would leave any predictions in doubt, especially when the length scale of device is reduced to microns. Fundamentally speaking, this calls for a knowledge of the non-equilibrium behavior of crystals. Overflow of energy from one crystal to another deficient in energy cannot be addressed using equilibrium continuum mechanics theories because they choose to assume the conditions across a surface or interface by letting $\Delta V / \Delta A \rightarrow 0$. This is the equivalent of assuming $R_{0} / 2$ to approach zero in Eq. (32). Equilibrium continuum mechanics considers only crystal size in the limit that approaches zero. Stated more precisely:

Classical continuum mechanics is not intended to address conditions across any surface or interface; it specifies them as boundary conditions. 
Computer simulation of atomic models could not supply the information if their solutions depend on specifying the conditions on the boundary. Strictly speaking, there prevail only bi-phase problems where the conditions across an interface are derived as unknowns rather than specified as knowns. Fracture mechanics can make no headway unless these issues are brought to light. There are too many unknowns in the chain of events, presently scaled as nano, micro, meso and macro. "Mesofracture mechanics" is the necessary first step of a long journey!

\section{References}

[1] T.Y. Wu, W.T. Chen, R.A. Pearson, D.T. Read (Eds.), Application of Fracture Mechanics in Electronic Package and Materials, EEP-vol. 11 and AMD-vol. 64, ASME Electrical and Electronic Package and Applied Mechanics Division, New York, 1995.

[2] W.T. Chen, D.T. Read (Eds.), Application of Fracture Mechanics in Electronic Package, AMD-vol. 222 and EEPvol. 20, ASME Applied Mechanics Division and Electrical and Electronic Package Division, New York, 1997.

[3] G.C. Sih, Thermomechanics of solids: nonequilibrium and irreversibility, J. Theor. Appl. Fract. Mech. 9 (3) (1988) 175-198

[4] G.C. Sih, in: S. Sienietyez, P. Salamon (Eds.), Some Basic Problems in Nonequilibrium Thermomechanics, Taylor \& Francis, New York, 1992, pp. 218-247.

[5] G.C. Sih, D.M. Chou, Nonequilibrium thermal/mechanical response of 6061 aluminum alloy at elevated temperature, J. Theor. Appl. Fract. Mech. 12 (1) (1989) 19-31.

[6] G.C. Sih, Some basic problems in fracture mechanics and new concepts, J. Eng. Fract. Mech. 5 (2) (1973) 365-377.

[7] A. Kelly, N.H. Macmillan, Strong Solids, third ed., Clarendon Press, Oxford, UK, 1986.

[8] G.C. Sih, Introduction to a series on mechanics of fracture, in: G.C. Sih (Ed.), Methods of Analysis and Solutions of Crack Problems, Noordhoff, Leiden, 1973, pp. IX-XII (Now Kluwer Academic Publishers, Boston).

[9] A.A. Griffith, The phenomena of rupture and flow in solids, Philos. Trans. Roy. Soc. London, Ser. A 221 (1921) 163-198.

[10] V.L. Popov, E. Kroner, On the role of scaling in the theory of elastoplasticity, J. Phys. Mesomech. 1 (1998) 103-112.

[11] M.F. Horstemeyer, M.I. Baskes, Atomistic finite deformation simulations: a discussion on length scale effects in relation to mechanical stresses, J. Eng. Mater. Technol. 121 (1999) 114-119.

[12] M.F. Horstemeyer, M.I. Baskes, S.T. Plimpton, Atomistic simulation of size scales and strain rate effects on plasticity, J. Metals (in press).
[13] V.E. Panin, Overview on mesomechanics of plastic deformation and fracture of solids, Theor. Appl. Fract. Mech. 30 (1) (1998) 1-11.

[14] P.V. Makarov, Character of localized deformation and fracture of solids at mesolevel, in: G.C. Sih (Ed.), Mesomechanics 2000: Role of Mechanics for Development of Science and Technology, vol. 1, Tsinghua University Press, 2000, pp. 143-152.

[15] G.C. Sih, Micromechanics associated with thermal/mechanical interaction for polycrystals, in: G.C. Sih (Ed.), Mesomechanics 2000: Role of Mechanics for Development of Science and Technology, vol. 1, Tsinghua University Press, 2000, pp. 143-152.

[16] G.C. Sih, Fracture mechanics of engineering structural components, in: G.C. Sih, L. Faria (Eds.), Fracture Mechanics Methodology, Martinus Nijhoff, Dordrecht, 1984, pp. 35-101 (Now Kluwer Academic Publishers, Boston).

[17] G.C. Sih, Mechanics of Fracture Initiation and Propagation, Kluwer Academic Publishers, The Netherlands, 1991.

[18] G.C. Sih, in: Mechanics of Fracture, vol. I-VII, Sijthoff and Noordhoff, The Netherlands, 1973-1981.

[19] G.C. Sih, C.T. Li, Subcritical crack growth in elasticplastic material with overshoot: Part I - analytical modelling, J. Theor. Appl. Fract. Mech. 18 (1993) 115-129.

[20] G.C. Sih, C.T. Li, Subcritical crack growth in elasticplastic material with overshoot: Part II - numerical computation, J. Theor. Appl. Fract. Mech. 18 (1993) 115-129.

[21] G.C. Sih, E. Czoboly, F. Gillemot (Eds.), Absorbed Specific Energy and/or Strain Energy Density Criterion, Akademiai Kiado, Budapest, Hungary, 1980.

[22] I.F. Gillemot, Criterion of crack initiation and spreading, J. Eng. Fract. Mech. 8 (1976) 239-253.

[23] V.S. Ivanova, Synergetic analysis of the strain energy density criterion of Sih-Gillemot, J. Theor. Appl. Fract. Mech. 11 (2) (1989) 103-108.

[24] D.Y. Tzou, G.C. Sih, Thermal/mechanical interaction of subcritical crack growth in tensile specimen, J. Theor. Appl. Fract. Mech. 10 (1) (1988) 59-72.

[25] G.C. Sih, D.Y. Tzou, Plastic deformation and crack growth behavior, in: G.C. Sih, A.T. Ishlinsky, S.T. Mileiko (Eds.), Plasticity and Failure Behavior of Solids, Martinus Nijhoff, Dordrecht, 1990, pp. 91-114 (Now Kluwer Academic Publishers, Boston).

[26] G.C. Sih, Fracture toughness concepts, in: Properties Related to Fracture Toughness STP 605, American Society of Testing and Materials, Philadelphia, PA, 1976, pp. 3-15.

[27] G.C. Sih, Mechanics of ductile fracture, in: G.C. Sih, C.L. Chow (Eds.), Fracture Mechanics and Technology, vol. 29, Sijthoff \& Noordhoff, Alphen aan den Rijn, 1997, pp. 767783.

[28] J.A. Kies, J.M. Kraft, R.J. Sanford, H.L. Smith, A.M. Sullivan, Historical note on the development of fracture mechanics, in: G.R. Irwin, G.C. Sih, R.P. Wei, F. Erdogan (Eds.), Linear Fracture Mechanics, Envo Publishing Co, Bethlehem, PA, 1974, pp. 1-27. 
[29] A. Tobin, Y.E. Pak, Effects if electric fields on fracture behavior of PZT ceramics, in: V.K. Varadan (Ed.), Smart Materials, vol. 1916, SPIE, 1993, pp. 77-86.

[30] Y.E. Pak, A. Tobin, On the electric field effects in fracture of piezoelectric materials, in: Mechanics of Electromagnetic Materials and Structures, AMD-vol. 161 and MDvol. 42, ASME, New York, 1993.

[31] H. Gao, T.Y. Zhang, P. Tong, Local and global energy release rates for an electrically yielded crack in a piezoelectric ceramic, J. Mech. Phys. Solids 45 (1997) 491-510.

[32] S. Park, C.T. Sun, Fracture criterion of piezoelectric ceramics, J. Am. Ceram. Soc. 78 (1995) 1475-1480.

[33] J.Z. Zuo, G.C. Sih, Energy density formation and interpretation of cracking behavior for piezoelectric ceramics, J. Theor. Appl. Fract. Mech. 34 (1) (2000) 17-33.

[34] G.C. Sih, J.Z. Zuo, Multiscale behavior of crack initiation and growth in piezoelectric ceramics, J. Theor. Appl. Fract. Mech. 34 (2) (2000) 123-141.

[35] K.J. Cheng, S.Y. Cheng, Boundary conditions of electrons at interface. Part I: Mixture of nanometer crystals and amorphous silicon, in: G.C. Sih, V.E. Panin (Eds.), Prospects of Mesomechanics in the 21st Century (special issue), J. Theor. Appl. Fract. Mech. 37 (2001) 11-17.

[36] K.J. Cheng, S.Y. Cheng, Boundary conditions of electrons at interface. Part II: Internal stresses in thin films, in: G.C. Sih, V.E. Panin (Eds.), Prospects of Mesomechanics in the 21 st Century (special issue), J. Theor. Appl. Fract. Mech. 37 (2001) 19-27.

[37] G.C. Sih, J.W. Ho, Sharp notch fracture strength characterized by critical energy density, J. Theor. Appl. Fract. Mech. 16 (3) (1991) 179-214.

[38] E. Orowan, Energy criterion of fracture, in: Welding Res. Suppl., 1955, pp. 157s-160s.

[39] G.C. Sih, C.K. Chao, Scaling of size/time/temperature associated with damage of uniaxial specimens - Part I: Progressive damage in uniaxial tensile specimen, J. Theor. Appl. Fract. Mech. 12 (2) (1989) 93-108.

[40] G.C. Sih, C.K. Chao, Scaling of size/time/temperature associated with damage of uniaxial specimens - Part II:
Progressive damage in uniaxial compressive specimen, J. Theor. Appl. Fract. Mech. 12 (2) (1989) 109-119.

[41] A.J. Rosakis, O. Samudrala, D. Coker, Intersonic shear crack growth along weak planes, Mater. Res. Innovat. 3 (1999) 236-243.

[42] A.J. Rosakis, O. Samudrala, D. Coker, Cracks faster than the shear wave speed, Science 284 (1999) 1337-1346.

[43] F.F. Abraham, H.J. Gao, How fast can cracks propagate?, Phys. Rev. Lett. 84 (14) (2000) 3113-3116.

[44] D.N. Fang, Fracture Mechanics Laboratory, Department of Engineering Mechanics, Tsinghua University, Beijing, China, April 2000, private communication.

[45] G.C. Sih, C. Chen, Non-self similar crack growth in an elastic finite thickness plate, J. Theor. Appl. Fract. Mech. 3 (2) (1985) 125-139.

[46] B.L. Holian, S.J. Zhou, P.S. Lomdahl, N. GronbechJensen, D.M. Beazley, R. Ravelo, Molecular-dynamics simulation of fracture: an overview of system size and other effects, Mater. Res. Soc. Symp. Proc. 409 (1996) 3-9.

[47] H. Noguchi, Y. Furuya, A method of seamlessly combining a crack tip molecular dynamics enclave with a linear elastic outer domain in simulating elastic-plastic crack advance, Int. J. Fract. 87 (1997) 309-329.

[48] Y. Furuya, H. Noguchi, A combined method of molecular dynamics with micromechanics improved by moving the molecular dynamics region successively in the simulation of elastic-plastic crack propagation, Int. J. Fract. 94 (1998) $17-31$.

[49] G.C. Sih, Z.F. Song, Dynamic analysis of tetragonal perovskite structure ceramics, implicated by asymptotic field solutions and boundary conditions, Theor. Appl. Fract. Mech. 38 (1) (2002).

[50] C. Sih, Dynamics crack problems - strain energy density fracture theory, in: G.C. Sih (Ed.), Mechanics of Fracture, vol. 4, Noordhoff, Leiden, 1997, pp. XVII-XLVII.

[51] M.E. Kipp, G.C. Sih, The strain energy density failure criterion applied to notched elastic solids, Int. J. Solids Struct. 2 (1975) 153-173. 